PPPL-3150 - Preprint: November 1995, UC-427

\title{
Theoretical Analysis of Driven Magnetic Reconnection Experiments
}

\section{Uzdensky, R. Kulsrud, and M. YamadaPrinceton Plasma \\ Physics Laboratory \\ P.O. Box 451 \\ Princeton, NJ 08543}

\begin{abstract}
DISCLAIMER
This report was prepared as an account of work sponsored by an agency of the United States Government. Neither the United States Government nor any agency thereof, nor any of their employees, makes any warranty, express or implied, or assumes any legal liability or responsibility for the accuracy, completeness, or usefulness of any information, apparatus, product, or process disclosed, or represents that its use would not infringe privately owned rights. Reference herein to any specific commercial product, process, or service by trade name, trademark, manufacturer, or otherwise does not necessarily constitute or imply its endorsement, recommendation, or favoring by the United States Government or any agency thereof. The views and opinions of authors expressed herein do not necessarily state or reflect those of the United States Government or any agency thereof.
\end{abstract}

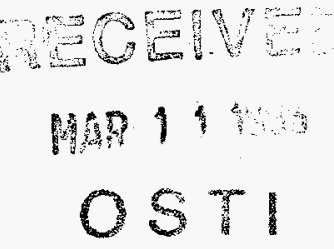




\section{NOTICE}

Page(s) size did not permit electronic reproduction. Information may be purchased by the general public from the National Technical Information Service, U.S. Department of Commerce, Springfield, VA 22161 (Area Code 703-487-4650). DOE and DOE contractors may purchase information by contacting DOE's Office of Scientific and Technical Information, P.O. Box 62, Oak Ridge, TN 37831, Attn: Information Services (Area Code 423-576-8401). 



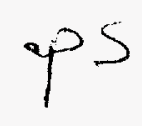

\title{
Theoretical Analysis of Driven Magnetic Reconnection Experiments.
}

\author{
Dmitri A. Uzdensky, Russell M. Kulsrud, and Masaaki Yamada \\ Princeton Plasma Physics Laboratory, P.O.Box 451, and Princeton University Observatory, \\ Princeton University, Princeton, New Jersey 08543
}

(August 22, 1995)

In this paper we develop a theoretical framework for the Magnetic Reconnection Experiment (MRX) in order to understand the basic physics of the experiment, including the effect of the external driving force, and the difference between co and counterhelicity cases of the experiment. In order to simplify the problem we reduce it to a 1-D resistive MHD model. Also, we define a special class of holonomic boundary conditions under which a unique sequence of global equilibria can be obtained, independent of the rate of reconnection. This enables us to break the whole problem into two parts: a global problem for the ideal region, and a local problem for the resistive reconnection layer. We carry out the calculations and obtain the global solution for the ideal region in one particular case of holonomic constraints, the so called "constant force" regime, for both the co and counterhelicity cases. After the sequence of equilibria in the ideal region is found, we tackle the problem of the rate of reconnection in the resistive reconnection region. This rate tells us how fast we proceed through the sequence of global equilibria but does not affects the sequence itself. Assuming the Sweet-Parker model for the reconnection layer, we calculate the reconnection rate, and demonstrate the difference between the co and counterhelicity cases, as well as the role of the external forces. We find our results to be in a reasonable agreement with the experiment. 


\section{INTRODUCTION}

Recently, experiments were performed [1-3] to explore the physics of magnetic reconnection. These experiments consisted of bringing together two spheromaks with common major axis. The toroidal currents in the two spheromaks were in the same direction. Thus, the poloidal fields in the region between the merging spheromaks were oppositely directed, so that magnetic reconnection of them could occur. The toroidal fields of the two spheromaks were parallel in one case of the experiments and antiparallel in another. These two cases were denoted as cohelicity and counterhelicity cases, respectively. The magnetic reconnection rate was appreciably faster in the counterhelicity case.

Since the magnetic reconnection phenomenon is of great importance, both in laboratory experiments [4-5] and in astrophysics (see [6], and references therein), it was decided to carry out more detailed experiments with larger spheromaks and with a more elaborate and controlled environment [7].

In order to understand the physics of these experiments we have carried out a simplified theory of the merging process. This theory attempts to describe the experiments on the basis of resistive magnetohydrodynamics in order to isolate how much of the phenomena can be understood without resort to finite gyration radius effects, anomalous resistivity and other more complicated effects. Since in the experiments the magnetic Reynolds number $R_{m}$ (or, strictly speaking, the Lundquist number $S$ ) is very large, we take $R_{m}>1$ throughout this paper.

We found that the general results of the experiments can be understood by such a description including the dependence of the reconnection rate on the external forces, and the gross differences in the reconnection rates between the co and counterhelicity cases. However, our model for the solution of the resistive magnetohydrodynamic equations is too crude to give very precise agreement.

Our paper appears in stages of increasing simplification.

The general situation during the reconnection process in the experiment can be seen in Figs. 1a and 1b. Fig. 1a indicates the two spheromaks just about to merge. Fig. 1b 
shows the situation after some of the poloidal magnetic flux has merged. In this Figure, regions I are the parts of the spheromaks that have not yet merged. We call these regions the unreconnected regions. They come from the regions labeled 1 in Fig. 1a. In Fig. 1b, region II is the common region of merged poloidal flux. We call this region the reconnected region. It comes from the regions labeled 2 in Fig. 1a. (At $t=0$ region II does not exist). In the cohelicity case this region also contains the toroidal flux that was originally in the regions 2 of Fig. 1a. In the counterhelicity case there is no toroidal flux in region II since, in this case, the toroidal flux is destroyed in the reconnection process. Finally, outside the spheromaks, there is a vacuum region, whose geometry is determined by external coils and walls.

Since the reconnection occurs on a time scale much shorter than the characteristic diffusion time, total poloidal magnetic flux in the plasma $\Psi_{t o t}$ remains unchanged. We take the external walls/coils to be infinitely conducting, so that the amount of poloidal magnetic flux in the vacuum region, $\Psi_{v a c}$, also remains unchanged during the reconnection process.

These external coils or infinitely conducting walls can provide some force, which pushes the two plasmas towards each other and squeezes them. As we shall see, this external force can accelerate the reconnection process, which allows us to call it the driving force. If there are no external boundaries (walls or coils), that is, if $F_{e x t}=0$, the reconnection is nondriven, and the developing current sheet balances only the force of mutual attraction of the two pinches.

We consider a situation where the toroidal magnetic field is produced only by the plasma currents, so that there is no toroidal magnetic field in the vacuum region.

At the midplane, a very narrow current layer develops between the two spheromaks, and the toroidal current in this layer provides the repulsive force, which balances the force of mutual attraction between the two spheromaks and the external pushing force. This layer is called the reconnection region, and its treatment requires resistive MHD, because of the very high current density. It is in this layer that the reconnection occurs and poloidal and, in the counterhelicity case, some of the toroidal magnetic field energy is converted into the 
plasma thermal and kinetic energy.

As the magnetic field lines merge, the plasma flows from region I into the reconnection layer, where it is joule heated. The resulting pressure gradient and magnetic tension accelerate the plasma outward along the layer up to about the Alfvén speed. Then, the plasma flows into the boundary region between regions I and II. This boundary region is expected to be very thin and we treat it as a singular magnetic surface. We call this surface the separatrix, because it separates regions with different topology of the poloidal magnetic field.

In the cohelicity case, the motions of plasma are purely poloidal. In the counterhelicity case, however, in addition to the poloidal motions, local toroidal flows are also produced, by the slingshot effect of the merging of the oppositely directed toroidal fields. Fig. 2 gives us a full three-dimensional picture of this process. The pairs of cylinders in (b) and (c) correspond to flux surfaces just prior to the reconnection. The cylinders in $\left(b^{\prime}\right)$ and $\left(c^{\prime}\right)$ are the same flux surfaces just after the reconnection. It is seen that in $\left(c^{\prime}\right)$ part of the toroidal field has been annihilated in the reconnection layer. Just after merging the toroidal lines have a sharp kink which accelerates the plasma in the toroidal direction. This motion leads to an Alfvén wave propagating in the separatrix region. The bulk of the toroidal field energy is converted into the energy of the Alfvén wave.

For the conditions of the experiment the motions in the separatrix region are damped out rapidly, compared to the rate of reconnection, so that this region is quite narrow. The poloidal motions are damped by the symmetry of the situations since the two flows emerging from the different sides of the resistive layer flow along the separatrix and collide at the top and bottom of it by symmetry. The resulting shock should damp these motions quickly. The toroidal motions associated with the Alfven wave in the separatrix region are damped by perpendicular viscosity. The damping of this wave is also rapid compared to the reconnection rate. As a consequence, as reconnection proceeds, a reconnected surface moves deeper into region II, but before it has moved very far, all the motions are damped. Therefore, motions exist only in the very thin separatrix region between I and II. 
Any plasma pressure in region I or II should evolve adiabatically as the magnetic merging continues. Also, both poloidal and toroidal fluxes are frozen in the plasma in both these regions, which allows us to call these two regions the ideal regions, and to describe them by the ideal MHD formalism. However, in the transition through reconnection, the entropy of the plasma should change, because the kinetic energy of the poloidal, and, in the counterhelicity case, the toroidal motions is converted into heat.

In this paper we show how the amount of conversion of magnetic energy to heat can be determined by the first law of thermodynamics. The work done by the external coils on the spheromaks during any time interval must be equal to the change in the total energy of the plasma in regions I, II, and in the vacuum.

Since there are no motions in regions I and II, these regions are in a magnetostatic equilibrium which is totally determined by the amount of poloidal flux in each region, the relation of the toroidal and poloidal fluxes, and the relation of the pressure to the poloidal flux. In region I these relations are given by flux freezing and the adiabatic evolution of the pressure. Similarly, after the plasma enters region II, these relations are given by the same conditions. The significant change is produced by the transfer of poloidal flux from region I to region II caused by reconnection. The amount of toroidal flux per unit of poloidal flux that enters region II is discussed above for the co and counterhelicity cases. The initial value of $P / \rho^{\gamma}$ on the freshly reconnected surface labeled by $\Psi_{s}$ (now entering region II) is given by equating the change in energy between two subsequent equilibria, to the work done.

In the special case where the position of the bounding walls is fixed in time, the subsequent equilibrium at $\Psi_{s}+\Delta \Psi_{s}$ is uniquely specified by the energy conservation condition. Hence, independent of what goes on in the reconnection layer, the entire sequence of equilibria in regions I and II can be determined.

It is clear that if our boundary conditions are more general than the fixed wall, but only involve the amount of unreconnected flux $\Psi_{s}$ in their statement, then one can find selfconsistently the new position of the wall, the new pressure profile, and the new equilibrium independently of time, or of the reconnection rate. Such boundary conditions clearly lead to 
a unique sequence of equilibria in the ideal regions. We designate such boundary conditions as holonomic conditions in analogy with time independent constraints in mechanics [8]. In our paper we present an example of holonomic conditions which is relevant to the experiment and different from the case of the fixed wall. We also discuss the holonomic and nonholonomic conditions in general in Section V of this paper.

Under the holonomic conditions, the only significant role of the reconnection region is to specify the rate at which the plasma proceeds through the uniquely given sequence of equilibria. This reconnection rate is determined by the boundary layer analysis of the reconnection region. It turns out that this analysis depends on the length of the layer, and on the variation of the outside magnetic field along the layer. These quantities are determined at any given time by the solutions in the ideal regions I and II.

To summarize, in the holonomic case the total problem can be broken into two separate analyses:

1) The global analysis of the ideal regions I and II. The unique sequence of magnetostatic equilibria is specified independently of the local physics of the reconnection region (e.g. the nature of the resistivity or the internal structure of the layer).

2) The local analysis of the reconnection region itself which determines the reconnection rate. This depends on the properties of the outer solution only just outside of the layer, as well as on the local physics of the layer.

Although this division of the whole problem into two separate ones does reduce the problem considerably, we are still left with a very complicated problem that can only be solved with any precision by a difficult numerical simulation. However, for the conditions of the experiment where the external force pushing the spheromaks together is strong, the spheromaks are considerably flattened. This allows us to simplify the geometry by taking regions I and II approximately one-dimensional (see Fig. 3). The validity of the onedimensional approximation can be easily seen if one looks only at the line of symmetry perpendicular to the reconnection layer. On this line the primary forces are those due to magnetic pressure and gas pressure. Because of the flattening the magnetic tension 
forces are considerably smaller. Thus, the equilibrium along this line is close to that of a one-dimensional equilibrium, and the behavior along this line well represents the total equilibrium as far as the rate of reconnection is concerned.

This one-dimensional problem is much more tractable than the complete two-dimensional problem. The magnetostatic equilibrium depends on the external forcing magnetic field just above and below the 1-D equilibrium, $B_{w}$. In general, $B_{w}$ varies with time due to changes in the geometry of the (now) "one-dimensional spheromaks" of our paper, and the changing external conditions. The 1-D problem becomes much simpler in the case where $B_{w}$ is a constant in time. This turns out to be a reasonable approximation to the driven reconnection experiment with constant external forcing, and is, in fact, a holonomic constraint. It is this case that we present in this paper.

In Section II we set up the equilibrium problem in the ideal regions. In II-A we derive the 1-D approximation, and in II-B we give the initial equilibrium. In section III we determine the general sequence of equilibria in the ideal regions. In III-A we derive the mass flux function, and in III-B the toroidal flux function. In III-C we give the equilibrium in terms of these flux functions and the yet unknown pressure function. In Section III-D we introduce the "constant $B_{w}$ "-approximation. In Section III-E we make use of these equilibrium results and energy conservation to determine the pressure function, and, thus, the actual sequence of equilibrium solutions through which the plasma progresses due to reconnection.

In Section IV we assume, for definiteness, that the reconnection rate is given by the physical model of Sweet-Parker. We show how it is modified by the presence of the toroidal field in the co and counterhelicity cases. In the framework of this model, we give the time evolution of the plasma. In Section $\mathrm{V}$ we discuss the significance of our results for general reconnection problems, and in Section VI we draw our conclusions. In the Appendix, we describe the conditions under which the assumption $B_{w}=$ const is reasonable. 


\section{MODEL OF THE IDEAL REGION.}

First, let us consider the solution in the ideal regions I (unreconnected or individual flux) and II (reconnected or common flux). Region I is symmetrical with respect to the magnetic axis, and the whole picture is symmetrical with respect to the midplane. Because of this overall symmetry with respect to the midplane, we can consider only the upper spheromak.

In each of regions I and II, plasma motions are slow (since the magnetic Reynolds number $R_{m} \gg>1$ ) compared with Alfvén speed, therefore, the solution in these two regions can be described by a sequence of magnetostatic equilibria with boundary conditions given by external coils or walls. This sequence depends on one free parameter $\Psi_{s}$, the amount of unreconnected poloidal flux. This parameter defines how far the plasma has progressed through the sequence of magnetostatic equilibria, and, thus, represents the slow evolution in time associated with reconnection: $\Psi_{s}(t=0)=\Psi_{t o t}$ is the total poloidal flux in plasma of one spheromak, measured from its magnetic axis, and $\Psi_{s}(t=\infty)=0$. The relationship between $\Psi$, and time, that is the rate at which the global equilibrium states follow each other, is called the reconnection rate, and is determined by the solution in the resistive reconnection region (see Section IV).

We describe the equilibrium solutions by representing the plasma parameters, such as pressure, density, poloidal and toroidal magnetic fields, etc., as functions of $\Psi$ and $\Psi_{s}$, where poloidal flux $\Psi$, measured from the magnetic axis, plays a role of a coordinate, and $\Psi_{s}$ plays a role of time variable. At any given moment of time (and, therefore, at any given value of $\left.\Psi_{s}\right)$ the relationship between the space coordinate $x$ and our new coordinate $\Psi$ is given by

$$
\vec{B}_{p o l}(\Psi)=\nabla \Psi \times \hat{z},
$$

where $\hat{z}$ is a unit vector in toroidal $(z)$ direction.

So, at each moment of time, plasma in regions I and II can be described by a solution of the MHD equilibrium equation:

$$
\vec{j} \times \vec{B}=\nabla P,
$$


which, itself, is totally determined once the four ideal region functions:

$$
P_{I}(\Psi), P_{I I}(\Psi), \Phi_{I}(\Psi), \Phi_{I I}(\Psi)
$$

- the plasma pressure and toroidal flux in regions I and II as functions of $\Psi$ - are given. These four functions are determined, in turn, by toroidal flux and energy conservation. For holonomic boundary conditions this determination is independent of the reconnection rate (see Section V).

\section{A. One-Dimensional Approximation.}

The general two-dimensional MHD equilibrium problem with a singular surface can be difficult, but it turns out that some major phenomena can be caught in the frameworks of a simple one-dimensional model.

Let us consider a situation where each plasma pinch (initially with circular structure of the flux surfaces), is squeezed in vertical ( $x$ ) direction by external coils and by the force of attraction with the other pinch. We shall call "strongly driven reconnection" the case when the mutual force is small compared to both the external force, and the repulsive force from the current layer. We consider only this case, so from now on the vertical size $a$ of each plasma is much smaller than its horizontal size $L$.

If $L_{0} \equiv L(t=0)>>a_{0} \equiv a(t=0)$, then we can represent the flux surfaces by ellipses or ovals (see Fig. 1a). In the middle of the process, that is, after some flux has been reconnected, the structure of the magnetic flux surfaces is shown in Fig. 1b.

The magnetic tension is small along the vertical $x$-axis, so, by assuming $L_{0}>>a_{0}$, and $L>>a$ ("driven reconnection"), we effectively make the problem one-dimensional. Thus, to simplify the situation, we model the problem by a rectangular geometry (see Fig. 3). We measure $x$-coordinate not from the midplane, but from the upper spheromak's magnetic axis which itself moves vertically during the reconnection process towards the midplane.

In this 1-D geometry, the external coils are represented by the infinitely conducting walls at the distance $x_{w}$ from the magnetic axes, that is at $x=x_{w}$ and at $x=-x_{w}-2 a$. We 
imagine these walls to be movable in order to control the external driving force. Region I, which appears to be disconnected in the 1-D model, is, actually, connected to itself, and region II ( $a<x<d$, where $d$ is the position of the plasma-vacuum boundary with respect to the magnetic axis) is connected to its reflection with respect to the midplane - region $[-a>(x+2 a)>-d]$. The separatrix magnetic surface is effectively connected to the reconnection region, because they are two parts of the same flux surface; during the reconnection, the mass from the lower part of this surface is added to the upper part. Similarly, in the cohelicity case, the toroidal fluxes are added, but, in the counterhelicity case, they cancel.

We describe the external pushing force in terms of the magnetic field in the vacuum region. This field is purely poloidal, and, in our 1-D geometry, it is uniform:

$$
B_{v a c}(x, t)=B_{w}(t)
$$

In general, we expect our 1-D approximation of the ideal region to be applicable, when $a, a_{0}<<L_{0}$ - "driven reconnection" regime, and $x_{w}<<L_{0}$, which can be satisfied by suitable geometry of the vacuum chamber, if the radius of the cylindrical chamber is much larger than its height. If $L_{0}>>x_{w}$, then, $L_{0}>>a_{0}$ is also satisfied, because $x_{w}>a_{0}$. Therefore, if we have a sufficiently "flat" vacuum chamber, then $L_{0}>x_{w}>a_{0}$, the reconnection process in this chamber will be "strongly driven", and we can use our 1-D model. However, even in the case where physical coils are further apart from each other than $2 L_{0}$, we believe that the vacuum field can be sufficiently well represented by walls that are much closer (at least, as far as the reconnection process itself is concerned).

\section{B. Initial equilibrium.}

For definiteness, let us choose a simple model for the initial equilibrium: let both the plasma density $\rho$ and the toroidal current density $j_{z}$ be uniform inside each plasma at $t=0$ :

$$
\rho=\rho_{0}=\text { const }, \quad \text { and } \quad j_{z}=\text { const },
$$


and let the initial equilibrium be force-free, $P_{0}=0$, so that the poloidal field pinch force is balanced by the toroidal field pressure.

Then, the poloidal flux as a function of $x$ is

$$
\Psi(x, t=0)=\alpha_{0} x^{2}, \quad x<a_{0},
$$

where

$$
\alpha_{0}=\frac{2 \pi}{c} j_{z}
$$

The initial poloidal magnetic field is

$$
B_{p o l}(t=0)=\frac{\partial \Psi(x, t=0)}{\partial x}=2 \alpha_{0} x=2 \sqrt{\alpha_{0} \Psi}=B_{w}(t=0) \sqrt{\frac{\Psi}{\Psi_{t o t}}},
$$

and the mass measured from the magnetic axis as a function of flux at $t=0$ is:

$$
M(\Psi)=\rho_{0} x=\rho_{0} \sqrt{\Psi / \alpha_{0}} .
$$

The toroidal field, which, in the case under consideration, is produced only by the plasma's poloidal currents, provides the force balance with poloidal field pressure gradient. Since the initial pressure is zero, we have:

$$
B_{p o l}^{2}(x, t=0)+B_{\text {tor }}^{2}(x, t=0)=\text { const }=B_{w}^{2}(t=0) .
$$

Then, if we use $B_{w}(t=0)=B_{p o l}\left(\Psi_{t o t}, t=0\right)=2 \sqrt{\alpha_{0} \Psi_{t o t}}$, we get:

$$
B_{t o r}^{2}=4 \alpha_{0}\left(\Psi_{t o t}-\Psi\right)=B_{w}^{2}(t=0)\left(1-\frac{\Psi}{\Psi_{t o t}}\right) .
$$

\section{SOLUTION FOR THE IDEAL REGION, $t>0$.}

\section{A. Mass conservation for $t>0$.}

In region $I, \Psi<\Psi_{s}$, magnetic field is frozen into the plasma, and mass as function of flux is conserved: 


$$
\rho_{I}\left(\Psi<\Psi_{s}\right)=\rho_{0} \frac{B_{p o l}\left(\Psi<\Psi_{s}\right)}{2 \sqrt{\alpha_{0} \Psi}}
$$

where, of course, $B_{p o l}\left(\Psi, \Psi_{s}\right)=\partial \Psi\left(x, \Psi_{s}\right) / \partial x$. (Thus, if the plasma compresses, $\rho$ increases as $B_{\text {pol. }}$ )

When $\Psi_{s}=\Psi$, that is at the moment of the reconnection of a particular flux surface, mass from the destroyed part of this flux surface is added to the upper part of the same surface (between regions I and II). Due to the symmetry of region I with respect to the magnetic axis, the plasma density and magnetic field on the two parts of the surface before reconnection are equal. Therefore, the amount of mass per flux on the upper part of the flux surface ( $\left.\Psi=\Psi_{s}, x=a\right)$ is doubled. After the reconnection of the given flux surface, the following evolution of plasma on this surface again obeys the frozen-in law. Then, in region $\mathrm{II}, \Psi>\Psi_{s}$, we have:

$$
\rho_{I I}\left(\Psi>\Psi_{s}\right)=\rho_{0} \frac{B_{p o l}\left(\Psi>\Psi_{s}\right)}{\sqrt{\alpha_{0} \Psi}}
$$

\section{B. Toroidal flux conservation.}

Since the characteristic reconnection time is small compared with the magnetic diffusion time, the toroidal magnetic flux $\Phi\left(\Psi, \Psi_{s}\right)$ (measured from the magnetic axis) is conserved separately in region $I$ and in region II. Then our analysis of the toroidal field evolution becomes similar to that of the density.

In region $I$, the result is:

$$
B_{t o r}\left(\Psi<\Psi_{s}\right)=B_{t o r}\left(\Psi, \Psi_{s}=\Psi_{t o t}\right) \frac{B_{p o l}\left(\Psi, \Psi_{s}\right)}{B_{p o l}\left(\Psi, \Psi_{t o t}\right)}
$$

or,

$$
B_{t o r}\left(\Psi<\Psi_{s}\right)=\sqrt{B_{w}^{2}\left(\Psi_{s}=\Psi_{t o t}\right)-4 \alpha_{0} \Psi} \frac{B_{p o l}\left(\Psi, \Psi_{s}\right)}{2 \sqrt{\alpha_{0} \Psi}}
$$

Since $\Psi_{s}$ plays a role of time, from now on we treat $B_{w}$ as a function of $\Psi_{s}$. 
In region II, that is after the reconnection, the situations for the co and counterhelicity differ. In the counterhelicity case toroidal magnetic field is completely destroyed:

$$
B_{\text {tor }}^{\text {counter }}\left(\Psi>\Psi_{s}\right)=0 \text {. }
$$

In the cohelicity case, the amount of toroidal flux containing on the given flux surface is doubled after the reconnection of the symmetrical flux surface, because all the toroidal flux from the destroyed part of the flux surface is added to the upper part of the same surface, just as it was for the density. Therefore,

$$
B_{t o r}^{c o}\left(\Psi>\Psi_{s}\right)=2 B_{t o r}\left(\Psi, \Psi_{t o t}\right) \frac{B_{p o l}\left(\Psi, \Psi_{s}\right)}{B_{p o l}\left(\Psi, \Psi_{t o t}\right)},
$$

or, to summarize,

$$
\begin{gathered}
B_{t o r}\left(\Psi<\Psi_{s}\right)=\sqrt{\Psi_{t o t} / \Psi-1} B_{p o l}\left(\Psi, \Psi_{s}\right) \\
B_{\text {tor }}^{c o}\left(\Psi>\Psi_{s}\right)=2 \sqrt{\Psi_{t o t} / \Psi-1} B_{p o l}\left(\Psi, \Psi_{s}\right) \\
B_{t o r}^{\text {counter }}\left(\Psi>\Psi_{s}\right)=0 .
\end{gathered}
$$

\section{Magnetostatic equilibrium.}

In our one-dimensional model, the condition of MHD equilibrium reduces to the vertical pressure balance equation and is very simple:

$$
P\left(\Psi, \Psi_{s}\right)+\frac{B_{p o l}^{2}\left(\Psi, \Psi_{s}\right)}{8 \pi}+\frac{B_{t o r}^{2}\left(\Psi, \Psi_{s}\right)}{8 \pi}=\frac{B_{w}^{2}\left(\Psi_{s}\right)}{8 \pi} .
$$

If we know functions $B_{t o r}\left(\Psi, \Psi_{s}\right)$, and $P\left(\Psi, \Psi_{s}\right)$ in each of the two ideal regions, then this equation is just an algebraic equation to determine $B_{p o l}\left(\Psi, \Psi_{s}\right)=\partial \Psi\left(x, \Psi_{s}\right) / \partial x$.

In region I for both co and counterhelicity $P=0$, and, thus, $B_{t o r}^{2}+B_{p o l}^{2}=B_{w}^{2}$. As we know, for $\Psi<\Psi_{s}, B_{t o r}^{2}=\left(\frac{\Psi_{\text {tot }}}{\Psi}-1\right) B_{p o l}^{2}\left(\Psi, \Psi_{s}\right)$, then, from the vertical pressure balance equation,

$$
B_{p o l}\left(\Psi, \Psi_{s}\right)=B_{w}\left(\Psi_{s}\right) \sqrt{\Psi / \Psi_{t o t}} .
$$


In region II pressure balance (20) can be written in dimensionless form as:

$$
\beta\left(\Psi, \Psi_{s}\right)+b^{2}\left(\Psi, \Psi_{s}\right)+b_{t o r}^{2}\left(\Psi, \Psi_{s}\right)=1
$$

where

$$
b \equiv \frac{B_{p o l, I I}}{B_{w}}, \quad b_{t o r} \equiv \frac{B_{t o r, I I}}{B_{w}}, \quad \beta \equiv \frac{8 \pi P_{I I}}{B_{w}^{2}} .
$$

We assume that the evolution of plasma on any particular flux surface is adiabatic during the entire reconnection process except for the short period of time when this particular surface is being reconnected. This means that evolution of plasma after the reconnection, i.e. in region II, obeys the adiabatic condition:

$$
\frac{P_{I I}\left(\Psi, \Psi_{s}\right)}{P_{I I}\left(\Psi, \Psi_{s}=\Psi\right)}=\left(\frac{\rho_{I I}\left(\Psi, \Psi_{s}\right)}{\rho_{I I}\left(\Psi, \Psi_{s}=\Psi\right)}\right)^{\gamma}=\left(\frac{B_{p o l, I I}\left(\Psi, \Psi_{s}\right)}{B_{p o l, I I}\left(\Psi, \Psi_{s}=\Psi\right)}\right)^{\gamma}
$$

\section{D. $B_{w}=$ const - approximation.}

The vacuum magnetic field $B_{w}$ as a function of time is essentially at our disposal. For the purpose of illustration, we consider the so called "constant force" or "constant $B_{w}$ " regime, which means that the position of the wall is constantly adjusted during the reconnection process (see the Appendix for details), so that $B_{w}(t)=$ const. This assumption will allow us to drastically simplify the mathematical procedures, making the problem much easier. In fact, this boundary condition is holonomic, as we will see.

If the position of the wall is fixed (which is more likely for a real experimental situation), the condition $B_{w}=$ const is also valid, if

$$
x_{w}>a_{0},
$$

because

$$
B_{w}=\frac{\Psi_{v a c}}{x_{w}-d}
$$

where $d(t) \sim a_{0}$ is the changing position of the plasma-vacuum boundary, and $\Psi_{v a c}$ is the magnetic flux between this boundary and the wall. If $x_{w}>>d \sim a_{0}$, then $B_{w}$ depends very weakly on $d$, and hence on $t$. 
Since plasma pressure evolves adiabatically in regions I and II, and since $B_{w}=$ const, nothing changes with time in these regions, except for the position of the separatrix between them. That is, in region I, the plasma pressure and magnetic fields as functions of $\Psi$ remain those of the initial equilibrium (the reason for this is that it is clearly impossible for the plasma and magnetic field to expand adiabatically without changing the total pressure, because the pressure of each of them would decrease). In region II, these functions are determined by the transition conditions at $\Psi=\Psi_{s}$.

Let us take a closer look at these regions.

In region I, Eq. (21) means that poloidal magnetic field, and, therefore, the plasma density and toroidal field remain unchanged throughout the reconnection process for both co and counterhelicity cases:

$$
\begin{gathered}
B_{p o l}\left(\Psi<\Psi_{s}\right)=2 \sqrt{\alpha_{0} \Psi} \\
\rho\left(\Psi<\Psi_{s}\right)=\rho_{0} \\
B_{t o r}\left(\Psi<\Psi_{s}\right)=2 \sqrt{\alpha_{0}\left(\Psi_{t o t}-\Psi\right)} .
\end{gathered}
$$

In region II, since $B_{w}=$ const, the solution is:

$$
\begin{gathered}
b\left(\Psi>\Psi_{s}\right)=b_{0}(\Psi) \equiv b\left(\Psi, \Psi_{s}=\Psi\right), \\
b_{t o r}\left(\Psi>\Psi_{s}\right)=b_{t o r, 0}(\Psi) \equiv b_{t o r}\left(\Psi, \Psi_{s}=\Psi\right),
\end{gathered}
$$

and

$$
\beta\left(\Psi>\Psi_{s}\right)=\beta_{0}(\Psi) \equiv \beta\left(\Psi, \Psi_{s}=\Psi\right),
$$

(here, subscript 0 indicates the moment of reconnection of the flux surface labeled by $\Psi$, i.e. $\left.\Psi_{s}=\Psi\right)$. This means that the magnetic field, density, and pressure stay unchanged in region II from the moment the given flux surface was reconnected until the end of the process, just as in region I. Thus, the only change of pressure, density, and magnetic fields as functions of $\Psi$ occurs at the moment $\Psi_{s}=\Psi$.

We see that in order to describe the equilibrium in region II, we need to find the three functions $b_{0}(\Psi), b_{t o r}(\Psi)$, and $\beta_{0}(\Psi)$ - the normalized poloidal and toroidal magnetic fields 
and pressure right after the reconnection. Therefore, we need three conditions for these three unknowns, and these conditions can be found by making use of the pressure balance, and two transition conditions at $\Psi_{s}=\Psi$ : toroidal flux conservation, and energy conservation.

Consider the reconnection of a very thin strip, or flux layer, $\left[\Psi_{1}, \Psi_{1}+\Delta \Psi\right]$ (see Fig. 4). Let $2 L$ be the length of this layer, $\Delta x$ and $\Delta x^{\prime}$ its width before and after the reconnection, and let $\delta \equiv \Delta x^{\prime} / \Delta x$. This dimensionless parameter $\delta\left(\Psi_{1}\right)$ is very important and represents the compression of plasma as a function of $\Psi_{1}$. Since the two regions of thickness $\Delta x$ are compressed into one region of thickness $\Delta x^{\prime}$, the actual compression of the plasma is $2 / \delta$.

Let us define the poloidal field $B_{0}$ just prior to the reconnection as

$$
B_{0}\left(\Psi_{1}\right)=B_{p o l, I}\left(\Psi_{1}, \Psi_{s}=\Psi_{1}\right)
$$

and

$$
\theta\left(\Psi_{1}\right) \equiv \frac{B_{0}}{B_{w}}=\sqrt{\frac{\Psi_{1}}{\Psi_{t o t}}}
$$

Then,

$$
B_{t o r, I}\left(\Psi_{1}\right) \equiv B_{t o r, I}\left(\Psi_{1}, \Psi_{s}=\Psi_{1}\right)=\sqrt{1-\theta^{2}} B_{w} .
$$

By flux conservation, the poloidal field just after the reconnnection is

$$
B_{0}^{\prime}\left(\Psi_{1}\right) \equiv B_{p o l, I I}\left(\Psi_{1}, \Psi_{s}=\Psi_{1}\right)=\frac{B_{0}}{\delta}=B_{w} \frac{\theta}{\delta},
$$

from which it follows that

$$
b_{0}(\theta)=\frac{B_{0}^{\prime}}{B_{w}}=\frac{\theta}{\delta(\theta)} .
$$

Then, using formulas (18)-(19) for the toroidal flux conservation,

$$
B_{t o r, I I}^{c o}=2 \frac{B_{t o r, I}}{\delta}=2 \frac{\sqrt{1-\theta^{2}}}{\delta} B_{w} \quad \Rightarrow \quad b_{t o r}^{c o}=2 \frac{\sqrt{1-\theta^{2}}}{\delta(\theta)}
$$

and

$$
B_{\text {tor, } I I}^{\text {counter }}=0 \quad \Rightarrow \quad b_{\text {tor }}^{\text {counter }}=0 .
$$

With this new notation, we can rewrite the pressure balance equation in region II right after the reconnection as follows: 
1) Cohelicity case:

$$
\beta_{0}+\frac{\theta^{2}}{\delta^{2}}+\frac{4\left(1-\theta^{2}\right)}{\delta^{2}}=1
$$

2) Counterhelicity case:

$$
\beta_{0}+\frac{\theta^{2}}{\delta^{2}}=1
$$

\section{E. Energy Conservation.}

We need to find an additional relationship between $\beta_{0}$ and $\delta$. Physically, $\beta_{0}$, that is, the pressure right after the reconnection, comes from the heating due to the dissipation of the motions in the separatrix region, as well as the compression produced by the external pressure $B_{w}^{2} / 8 \pi$. Rather than attempt to calculate the dissipation directly, which is very difficult, we simply make use of the fact that the change in total energy is only due to the work done by the external forces.

Therefore, we shall use the energy conservation requirement in order to find the additional relationship between $\beta_{0}(\theta)$ and $\delta(\theta)$.

Let us compare the total energy of plasma and magnetic field in the region between the midplane and the upper part of the $\Psi_{1}+\Delta \Psi$-surface before and after the reconnection of this surface.

Here, when we talk about "just before or just after the reconnection of a given flux surface" we imply that the time interval $\Delta t$ between these two moments is much shorter than the total reconnection time $\tau_{\text {rec }}$, but it is still much longer than the time of damping of all of the MHD motions on this particular surface. Then, at the moments "just before" and "just after" the reconnection of the given flux surface, this surface is in quasi-equilibrium with the neighboring surfaces, and its kinetic energy is negligible compared with the magnetic and thermal energy. Between these two moments, however, part of the magnetic field energy is temporarily converted into the kinetic energy of plasma moving with Alfvén speed out of the reconnection region in $y$-direction.

Let us define, in Fig. 4a, region $\mathrm{I}^{*}, \Psi<\Psi_{1}$ (i.e. $-a<x<a$ ), as region I without the 
flux strip under consideration. This region is identical to region I in Fig. 4b. Similarly, let us define, in Fig. $4 \mathrm{~b}$, region $I I^{*}, \Psi>\Psi_{1}+\Delta \Psi$, as region II without the flux strip under consideration. This region is identical to region II in Fig. 4a.

Since $B_{w}=$ const, region $I^{*}$ remains, as we already know, unchanged, it just sinks down to the midplane, and becomes region I in Fig. $4 \mathrm{~b}$. Therefore, its energy before the reconnection of the given flux layer is equal to its energy after the reconnection, and we can omit it from our energy balance.

Thus, we only need to calculate the total energy in the very thin strip, or flux layer, $\left[\Psi_{1}, \Psi_{1}+\Delta \Psi\right]$ before and after its reconnection, and the work done by the external forces. Before the reconnection of this flux layer plasma pressure in it is zero, so the total energy is just equal to the magnetic energy:

$$
E_{\text {before }}=2 \cdot 2 L \cdot \Delta x \frac{B_{\text {pol }}^{2}+B_{\text {tor }}^{2}}{8 \pi}=4 L \Delta x \frac{B_{w}^{2}}{8 \pi} .
$$

After the reconnection we have:

$$
E_{\text {after }}=2 L \Delta x^{\prime}\left[\frac{B_{p o l}^{2}+B_{t o r}^{2}}{8 \pi}+\frac{3}{2} P\right] .
$$

(Here we take $\gamma=5 / 3$.)

But the total pressure is constant and equal to $B_{w}^{2} / 8 \pi$, so $\frac{B_{p o l}^{2}+B_{\text {tor }}^{2}}{8 \pi}+\frac{3}{2} P=\frac{B_{w}^{2}}{8 \pi}+\frac{1}{2} P$, then

$$
E_{a f t e r}=2 L \Delta x \delta \cdot \frac{B_{w}^{2}}{8 \pi} \cdot\left[1+\frac{1}{2} \beta_{0}\right] .
$$

The flux strip under consideration changes its width by $\Delta x^{\prime}-2 \Delta x$, so that the work done by the outer (i.e. $\Psi>\Psi_{1}+\Delta \Psi$ ) flux surfaces is

$$
W=2 L\left(2 \Delta x-\Delta x^{\prime}\right) \frac{B_{w}^{2}}{8 \pi},
$$

because the total pressure is $B_{w}^{2} / 8 \pi$. 
Then the energy conservation relation

$$
E_{\text {after }}=E_{\text {before }}+W
$$

gives us the simple relationship:

$$
\beta_{0}=\frac{4(2-\delta)}{\delta} .
$$

This formula is valid for both co and counterhelicity. It must be combined with the pressure balance equations (40)-(41) to give us $\delta$ and $\beta_{0}$ as functions of $\theta=B_{0} / B_{w}=$ $\sqrt{\Psi / \Psi_{\text {tot }}}$.

For the cohelicity case we have:

$$
\delta^{c o}(\theta)=\frac{1}{5}\left(4+\sqrt{16+5\left(4-3 \theta^{2}\right)}\right) .
$$

For the counterhelicity case:

$$
\delta^{\text {counter }}(\theta)=\frac{1}{5}\left(4+\sqrt{16+5 \theta^{2}}\right) .
$$

Functions $\delta(\theta)$ and $\beta_{0}(\theta)$ are presented in Figs. 5 and 6 .

As we see, at the beginning of the process, $\theta=1$,

$$
\delta^{c o}(1)=\delta^{\text {counter }}(1)=\frac{1}{5}(4+\sqrt{21}) \simeq 1.7165 . .,
$$

and

$$
\beta_{0}^{c o}(1)=\beta_{0}^{\text {counter }}(1) \simeq 0.66 \ldots
$$

As the reconnection advances, $\delta$ increases in the cohelicity case, and, finally,

$$
\delta^{c o}(\theta=0)=2, \quad \text { and } \beta_{0}^{c o}(\theta=0)=0
$$

In the counterhelicity case, $\delta$ decreases with time, and

$$
\delta^{\text {counter }}(\theta=0)=1.6, \quad \text { and } \beta_{0}^{\text {counter }}(\theta=0)=1
$$

At this point we know everything about regions I and II. The solution in region $I$ is given by the initial configuration ( formulas (27)-(29)). In region II, $B_{p o l}=b_{0}(\theta) B_{w}, B_{t o r}=$ 
$b_{t o r}(\theta) B_{w}$, and $P_{I I}=\beta_{0}(\theta) \frac{B_{w}^{2}}{8 \pi}$ are determined by Eqs. (37), (38)-(39), and (47), respectively. All these equations involve $\delta$ and $\theta$, and $\delta(\theta)$ is given, for the co and counterhelicity cases, by Eqs. (48)-(49). Thus, all the plasma parameters characterizing the MHD equilibria in regions $I$ and II are specified in terms of fixed functions of $\theta=\sqrt{\Psi / \Psi_{\text {tot }}}$. These functions do not depend explicitly on the current value of $\Psi_{s}$ which changes as reconnection proceeds. The only way $\Psi_{s}$ affects the equilibrium is in giving the extent in $\theta$ for each region: the range of $\theta$ 's in region I $\left(0<\theta<\sqrt{\Psi_{s} / \Psi_{t o t}}\right)$ decreases, and the range of $\theta$ 's in region II $\left(\sqrt{\Psi_{s} / \Psi_{t o t}}<\theta<1\right)$ increases with time.

These equations give us the desired unique sequence of equilibria as a function of $\Psi_{s}$. It is clear that for different reconnection rates we pass through identical sequence of equilibria. The reason why this sequence is unique can be traced to our holonomic constraint $B_{w}=$ const. To show that it is holonomic, we present the following argument. At any particular $\Psi_{s}$, for the reconnection of a small amount of flux $\Delta \Psi$, the displacement of the plasmavacuum boundary, and, therefore, of the position of the wall, is $2 \Delta x-\Delta x^{\prime}=\Delta x(2-\delta)$ (from the condition $B_{w}=$ const and Eq. (26) it follows that $x_{w}(t)-d(t)$ is a constant). Since we know $\delta(\theta)$ in terms of our equilibrium calculation, we can then express our changing boundary conditions (i.e. position of the wall) directly in terms of a particular dependence $x_{w}\left(\Psi_{s}\right)$, which is completely independent of the reconnection rate (for more details, see the Appendix).

\section{RECONNECTION RATE.}

As we have mentioned before, the rate at which global MHD equilibria follow each other, is determined by the local solution in the very thin reconnection layer.

The detailed physics of the reconnection layer is not the purpose of this study (for good general reviews of various reconnection theories see [9]-[10]), so for the purpose of illustration, we use a modification of the simplest Sweet-Parker model for the reconnection of an incompressible plasma [11,12]. This model contains the physics necessary for the 
demonstration of the influence of the external forces on the reconnection rate, and of the difference between co and counterhelicity cases. In this Section we return to the general 2-D problem, but make use of some of the 1-D results of Section III.

According to usual 2-D Sweet - Parker theory [11]-[12], the reconnection rate for an incompressible plasma can be described by

$$
\dot{\Psi}_{s}=-V_{S P} B_{0}\left(\Psi_{s}\right)
$$

where $B_{0}\left(\Psi_{s}\right)$ is the poloidal magnetic field just outside the layer in region $I$, and the SweetParker reconnection velocity $V_{S P}$ is given by

$$
V_{S P}=\sqrt{\frac{\nu u}{L\left(\Psi_{s}\right)}}
$$

Here, $\nu=\frac{\eta c^{2}}{4 \pi}, \eta$ is the resistivity, $u$ is the speed of the plasma moving out of the layer in the poloidal direction, and $L\left(\Psi_{s}\right)$ is the layer length.

We emphasize the fact that the solution in the reconnection region depends on both local parameters of the layer and on the global solution: both $B_{0}$ and $L$ are determined by the ideal region solution.

One important effect we address here is the difference in the reconnection rates for co and counterhelicity cases. One reason for this difference can be attributed to the different values of the outward velocities $u$, and can be described as follows.

In the center of the reconnection region, $x=0, y=0$, at least, part of the magnetic field is destroyed (in this section we measure $x$ from the midplane). The magnetic energy is converted into the plasma pressure by joule heating. The pressure $P$ in the resistive reconnection region is determined by the vertical pressure balance. The plasma is expelled out of the reconnection region by both the pressure gradient and the magnetic tension. In a simple 2-D case with no toroidal field, these two forces are in the same poloidal direction (i.e. along horizontal $y$-axis), and result in the total acceleration of plasma up to the Alfvén speed:

$$
u=V_{A} \equiv B_{0} / \sqrt{4 \pi \rho_{0}}
$$


where $\rho_{0}$ is the plasma density (we treat plasma inside the reconnection layer as an incompressible fluid).

In the cohelicity case, since the plasma is incompressible, the toroidal magnetic field is uniform. Therefore, it drops out of the fluid equations, and the above formula for $u$ is valid with $B_{0}=B_{p o l}$.

In the counterhelicity case, however, the situation is different. From the pressure balance, we have

$$
P^{\text {counter }}(0,0)=\left(B_{0}^{2}+B_{t o r}^{2}\right) / 8 \pi,
$$

which is larger than $P^{c o}(0,0)=B_{0}^{2} / 8 \pi$ by the toroidal magnetic field pressure. The pressure gradient is directed strictly along $y$-axis, but the magnetic tension force is directed along the magnetic field, because toroidal field is destroyed in the reconnection region just as the poloidal field is. The toroidal component of this magnetic tension force provides toroidal acceleration up to some velocity $v_{t o r}$. This has been observed in the experiments [1-3].

Then, we can write:

$$
v_{\text {tor }}^{2}+u_{\text {counter }}^{2}=V_{A w}^{2}
$$

where $V_{A w}=\frac{B_{w}}{\sqrt{4 \pi \rho_{0}}}$ - is Alfvén speed defined in terms of total magnetic field just outside the reconnection region, $B_{w}=\sqrt{B_{\text {tor }}^{2}+B_{0}^{2}}$.

Now, an important question arises: what is the relative importance of the magnetic curvature acceleration compared with the acceleration due to the pressure gradient? To answer this question, we would have to look deeper into the details of the reconnection region (i.e. current layer), but, since this is not the primary subject of this paper, let us just assign some values $\sigma_{p}$ and $\sigma_{m}$ to the "relative importance" of acceleration due to pressure gradient and due to magnetic tension, respectively, $\sigma_{p}+\sigma_{m}=1$. Then we can write:

$$
u_{\text {counter }}^{2}=V_{A w}^{2}\left(\sigma_{p}+\theta^{2} \sigma_{m}\right)
$$

and,

$$
v_{t o r}^{2}=V_{A w}^{2}\left(1-\theta^{2}\right) \sigma_{m},
$$


where $\theta=B_{0} / B_{w}$, as usual.

We can also rewrite the expression for $u^{c o}$ in these terms: $P^{\infty o}(0,0)=B_{0}^{2} / 8 \pi=\theta^{2} B_{w}^{2} / 8 \pi$, then:

$$
u_{c o}^{2}=V_{A w}^{2} \theta^{2}\left(\sigma_{p}+\sigma_{m}\right)=V_{A}^{2} .
$$

The difference between co and counterhelicity is now clear. The magnetic tension part of acceleration in $y$-direction is provided, in both cases, by the poloidal field only: the $V_{A w}^{2} \theta^{2} \sigma_{m}$ - term is the same in formulas (59) and (61), but the pressure gradient acceleration in the counterhelicity case is greater than in the cohelicity case. This is because, in the counterhelicity case, the total magnetic field energy $B_{w}^{2} / 8 \pi$ is converted into the plasma pressure in the reconnection region, whereas, in the cohelicity case, the toroidal magnetic field remains unchanged and uniform, and the plasma pressure in the reconnection region is equal to the poloidal field pressure just outside the layer.

Since at the beginning of the process the toroidal field is relatively weak, this difference is not important for small $t$. However, as reconnection advances, the toroidal field becomes stronger, the poloidal field becomes weaker, and this difference becomes very important.

Using the above expressions (59) and (61) we can now calculate the reconnection rate for co and counterhelicity cases, based on these simple Sweet-Parker-like arguments. Expression (55) is valid for both co and counterhelicity cases. The difference between the two cases comes from the different dependence of $u$ on $\Psi_{s}$.

In (54)-(55), $B_{0}$ and $L$ must be determined by the ideal region solution. From (27),

$$
B_{0}\left(\Psi_{s}\right)=B_{p o l}\left(\Psi=\Psi_{s}\right)=2 \sqrt{\alpha_{0} \Psi_{s}},
$$

As for the length of the layer, $2 L$, it is not really determined in the 1-D analysis of Section III. In the real 2-D problem this length changes with time, and, hence, it depends on $\Psi_{s}$. At any moment of time, we can evaluate the length of the reconnection region as being approximately equal to the length of the flux surface currently being reconnected. Assuming that all flux surfaces have the same shape (but different sizes), we can write the relationship between the length (i.e. size in $y$-direction) and the height (i.e. size in 
$x$-direction) of a flux surface:

$$
\frac{2 L(\Psi)}{2 x(\Psi)}=\frac{L_{0}}{a_{0}}=\text { const }
$$

independent of $\Psi_{\mathrm{s}}$.

Then, the length of the flux surface $\Psi_{s}$, and, therefore, of the reconnection layer, is given by:

$$
L\left(\Psi_{s}\right)=L_{0} \sqrt{\frac{\Psi_{s}}{\Psi_{t o t}}}
$$

(Here we used Eq. (6) for $x(\Psi)$.)

Substituting Eqs. (59) and (61) for $u$ in the co and counterhelicity cases, and formulas (62) and (64) into the general expression for $\dot{\Psi}_{s}$, we get simple first order differential equations for $\Psi_{s}(t)$ :

$$
\dot{\Psi}_{s}^{c o}=-B_{w}^{3 / 2} \sqrt{\frac{\nu}{L_{0} \sqrt{4 \pi \rho_{0}}}}\left(\frac{\Psi_{s}}{\Psi_{t o t}}\right)^{1 / 2}
$$

and,

$$
\dot{\Psi}_{s}^{\text {counter }}=-B_{w}^{3 / 2} \sqrt{\frac{\nu}{L_{0} \sqrt{4 \pi \rho_{0}}}}\left(\frac{\Psi_{s}}{\Psi_{t o t}}\right)^{1 / 4},
$$

with initial condition $\Psi_{s}(t=0)=\Psi_{t o t}$. (Since the values of $\sigma_{p}$ and $\sigma_{m}$ are uncertain, we take, for simplicity, $\sigma_{p}=1, \sigma_{m}=0$.)

The solution of these equations gives us the relationships between $\Psi_{s}$, the amount of unreconnected flux, and time $t$ for the co and the counterhelicity cases:

$$
\begin{gathered}
\Psi_{s}^{c o}(t)=\Psi_{t o t}[1-t / \tau]^{2}, \\
\Psi_{s}^{\text {counter }}(t)=\Psi_{t o t}\left[1-\frac{3}{2} \frac{t}{\tau}\right]^{4 / 3},
\end{gathered}
$$

where

$$
\tau=\sqrt{\frac{8 L_{0} \sqrt{\pi \rho_{0}} \Psi_{\text {tot }}^{2}}{\nu B_{w}^{3}}}=\sqrt{\frac{L_{0} \sqrt{\pi \rho_{0}} a_{0}^{3}}{\nu \Psi_{\text {tot }}}}=\sqrt{\frac{2 L_{0} \sqrt{\pi \rho_{0}} a_{0}^{2}}{\nu B_{w}}}
$$

is the characteristic reconnection time. Functions (67) and (68) are represented in Fig. 7.

Now, the external force $F$ (per unit area) driving the two spheromaks together is

$$
F \equiv \frac{B_{w}^{2}}{8 \pi}
$$


and we see that ( for fixed values of $\rho_{0}, \Psi_{t o t}, \nu$, and $L_{0}$ ) the reconnection rate $\gamma \equiv 1 / \tau$ varies with $F$ as

$$
\gamma \sim F^{3 / 4}
$$

This is in a qualitative agreement with the experiments [1-3].

A typical set of parameters relevant for the experiments $[1-3]$ is: $T=10 \mathrm{eV}, B_{w}=10^{3} \mathrm{G}$, $n=10^{14} \mathrm{~cm}^{-3}$, and $L_{0}=a_{0}=10 \mathrm{~cm}$, therefore, it is convinient to write Eq. (69) in the following form:

$$
\tau \simeq 12 \mu \sec \left(\frac{T}{10 \mathrm{eV}}\right)^{3 / 4}\left(\frac{B_{w}}{1 k G}\right)^{-1 / 2}\left(\frac{n}{10^{14} \mathrm{~cm}^{-3}}\right)^{1 / 4}\left(\frac{L_{0}}{10 \mathrm{~cm}}\right)^{1 / 2}\left(\frac{a_{0}}{10 \mathrm{~cm}}\right)
$$

As we see, the total reconnection time for the counterhelicity case is somewhat shorter than the total reconnection time for the cohelicity case, and the difference in rates is largest toward the end of the process. These results also agree with the experiments [1-3].

In this Section we have assumed the Sweet-Parker model for the reconnection region. Employing the same parameters $B_{0}$ and $L$ derived from the ideal region solution, other reconnection models (such as [13]-[17]) could have been assumed.

\section{GENERAL ASPECTS OF RECONNECTION.}

We have employed a one-dimensional approximation to understand the physics of the magnetic reconnection experiments. Although this approximation is an oversimplification which is not expected to yield great precision, it has many ingredients in common with a more general approach to reconnection. To demonstrate this, let us review our problem in a more general context.

As reconnection progresses, the global region passes through a sequence of equilibria. In our 1-D case, the equilibria are specified in terms of the position of the wall with respect to the midplane $X_{w}=x_{w}+a$, the amount of unreconnected flux $\Psi_{s}$, the pressure as a function of $\Psi, P(\Psi)$, and the toroidal field as a function of $\Psi, B_{t o r}(\Psi)$. Since these functions evolve as we go from one equilibrium to another, we can label the members of the sequence by $\Psi_{s}$, so 
that the whole sequence of equilibria is determined as long as functions $X_{w}\left(\Psi_{s}\right), P\left(\Psi, \Psi_{s}\right)$, and $B_{\text {tor }}\left(\Psi, \Psi_{8}\right)$ are known.

We have given a prescription for how to advance the toroidal field and pressure functions with $\Psi_{\text {a }}$ (as reconnection progresses). Namely, the evolution of $B_{\text {tor }}$ obeys the frozen-in law in the ideal region, and the change at the reconnection point is obtained by simply adding or canceling toroidal fluxes. $P\left(\Psi, \Psi_{s}\right)$ changes adiabatically away from the reconnection region, and on the newly reconnected surface it is chosen according to the 1st law of thermodynamics. That is the work done by the change in the wall position, $\Delta W=2 L \cdot \Delta X_{w} \frac{B_{w}^{2}}{8 \pi}$, is equal to the difference of the total energies just before and after the reconnection of the given flux layer (in fact, this is the work done upon only one of the two spheromaks, so that the total work is twice this amount).

Now the question is how to incorporate the possible evolution of the boundary conditions into the whole picture. In this paper, we chose, for illustration, a particular evolution of the boundary conditions, namely, "constant force" $B_{w}=$ const. This regime requires continuous adjusting of the position of the wall which can be expressed by the particular dependence of $X_{w}\left(\Psi_{s}\right)$, given by expressions (A5)-(A6) from the Appendix. Because of this, we are able to specify the functions characterizing the equilibrium, i.e. $X_{w}\left(\Psi_{s}\right), P\left(\Psi, \Psi_{s}\right)$, and $B_{t o r}\left(\Psi, \Psi_{s}\right)$ independently of the reconnection rate, and, therefore, of the local physical processes in the reconnection region. These processes include, for example, the nature of the resistivity (Spitzer or anomalous), FLR-effects, and the internal structure of the reconnection layer (i.e. that of Sweet-Parker [11]-[12], or of Petschek [13]). This enables us to completely break the problem into two separate problems, a global, and a local one. Thus, for example, if the resistivity in the reconnection region were anomalous, we would go through exactly the same sequence of global equilibria as we would if the resistivity were Spitzer (only the rate would be different), and the same is true for Sweet-Parker vs. Petschek. In general, this break up is possible whenever the boundary conditions are tied explicitly to $\Psi_{s}$ in any way, for example, if $X_{w}$ is a constant in time (position of the wall is fixed).

On the other hand, if the boundary conditions are nonholonomic, i.e. $X_{w}$ is given as 
a general function of time, the break up is not possible, since the work done is no longer uniquely expressible independently of the reconnection rate. The sequence of global equilibria would depend strongly on the local physics of the reconnection layer.

Let us try to understand how we would derive the sequence of global equilibria in this nonholonomic case, given all the information we need about the reconnection region. After we specify the physics of the layer, the reconnection rate $\dot{\Psi}_{s}$ could be expressed as a function of several global parameters such as the magnetic field just outside the layer $B_{0}$, and the length of the layer $L$, i.e. $\dot{\Psi}_{s}=f\left(L, B_{0}\right)$. The particular form of the function $f$ depends on the resistivity in the layer and its internal structure, and, thus, contains all the information we need to know about the reconnection layer. With this function, it is possible to couple $X_{w}$ and $\Psi_{s}$ and consequently find the sequence of global equilibria, because $L$ and $B_{0}$ are given by the solution of the global equilibrium problem. Now, if $f\left(L, B_{0}\right)$ were given, for example, by the Sweet-Parker theory, the resulting sequence of equilibria would be quite different from the sequence obtained if $f\left(L, B_{0}\right)$ were given by the Petschek theory. The reason for this is that for any given value of $\Delta \Psi_{s}$, the change in the position of the wall, $\Delta X_{w}=\frac{1}{f\left(L, B_{0}\right)} \frac{d X_{w}}{d t} \Delta \Psi_{s}$, would be much smaller in the Petschek case, since the reconnection rate is faster. The difference in $\Delta X_{w}$ would result in the difference in the work done, and therefore, the pressure and current profiles would be completely different. This, in turn, would result in different values of $B_{0}$ and $L$, thus making the difference between the two cases even more extreme.

Thus, as far as the time evolution of the system is concerned, there are two aspects of the problem:

(1) the variation of the boundary conditions with time.

(2) the amount of reconnection that occurs in time.

In our simple one-dimensional model (1) is represented by only one controlling parameter $X_{w}$, and (2) by only one parameter $\Psi_{s}(t)$. Of course, any realistic 2-D or 3-D problem is much more complicated, and $X_{w}$ and $\Psi_{s}$ become continua of conditions which would represent a more general formalism. (For example, in the case of the solar corona, the adequate analog 
of $X_{w}$ is the mapping of the footpoints on the solar surface.) However, the physical essence of reconnection problems does seem to be captured by these two parameters of our simplified analysis.

The time evolution of a reconnecting system with changing boundary conditions can be expressed by a trajectory in $\left(\Psi_{s}, X_{w}\right)$-space. In Fig. 8 , we present trajectories for two particular cases of holonomic boundary conditions. Trajectory (a) corresponds to $X_{w}=$ const, and trajectories (b) and ( $\left.\mathrm{b}^{\prime}\right)$ correspond to $B_{w}=$ const in the co and counterhelicity cases, respectively. Here, $B_{w}$ and $X_{w}$ are related by

$$
B_{w}=\frac{\Psi_{v a c}}{X_{w}\left(\Psi_{s}\right)-d\left(\Psi_{s}\right)-a\left(\Psi_{s}\right)}
$$

(The amount of poloidal flux in the vacuum region, $\Psi_{v a c}$, is constant.)

In a rather broad class of cases, including $X_{w}=c o n s t$ and $B_{w}=c o n s t$, the amount of reconnected flux itself regulates the evolution of the boundary conditions. In these holonomic cases, the trajectory $X_{w}\left(\Psi_{s}\right)$, and, therefore, the whole sequence of magnetostatic equilibria in the ideal region, can be found explicitly without any knowledge about the physical processes in the reconnection layer. This, in a way, is analogous to shocks in hydrodynamics where the connection between upstream and downstream regions is independent of the physics inside the shock.

On the other hand, in many cases the time evolution of the boundary conditions can be arbitrary and totally independent of the amount of reconnected flux. Then, $X_{w}$ can only be given externally as a particular function of time. For example, in the case of solar corona, the adequate analog of $x_{w}$ is be the position of the footpoints, and, since the motion of the footpoints is not affected by the reconnection process above the solar surface, it is equivalent to giving the evolution of the boundary conditions in terms of the dependence $X_{w}(t)$, not $X_{w}\left(\Psi_{s}\right)$.

In these nonholonomic cases, in order to find $X_{w}\left(\Psi_{s}\right)$, and, therefore, the whole sequence of equilibria, it it essential to know the ratio between $X_{w}$ and $\dot{\Psi}_{s}$. That is, we need to know the actual reconnection rate as the function $f\left(L, B_{0}\right)$, which means that the sequence of 
equilibria depends strongly on the nature of the resistivity and on the physical internal structure of the reconnection region.

The above remarks about one-dimensional equilibria also apply to two-dimensional equilibria. The only major difference is that $X_{w}$ is now a function of poloidal angle $\theta$. As in the 1-D case, in the case of two dimensions each particular equilibrium is uniquely determined by the boundary conditions (represented now by $X_{w}(\theta)$ ), and by essentially the same two one-dimensional functions of $\Psi$, the plasma pressure $P(\Psi)$, and the toroidal flux $\Phi(\Psi)$. Again, if the conditions determining the evolution of the boundary are holonomic, so that one can specify the work done as $\Psi_{s}$ changes independently of the reconnection rate, then the sequence of 2-D equilibria is also independent of the reconnection rate. If the evolution of the boundary conditions is nonholonomic and given externally as a function of time (and $\theta !)$, then, in order to find the sequence of equilibria, one needs to know the reconnection rate as a particular function of $B_{0}$ and $L$.

The generalization to 3-D geometry is, apparently, more complicated, and we are uncertain whether analogous results can be obtained.

\section{CONCLUSIONS.}

The goal of this paper was to develop a theoretical framework to understand the physics of the MRX experiment. We found that this can be done by means of a simple one-dimensional model which is both tractable and leads to some valuable insights into the experiment.

We believe that some basic features of the merging process of two spheromaks, like dependence of the reconnection rate on the external forces, and the difference between co and counterhelicity cases, can be understood on the basis of a simple resistive MHD model.

The whole problem can be broken up into two separate problems:

- global solution for the ideal region,

- local solution for the resistive reconnection layer.

Under certain boundary conditions which we call holonomic $\left(B_{w}=\right.$ const is one example 
of such boundary conditions), plasma behavior in the ideal regions can be described by a sequence of magnetostatic equilibria, each of them being independent of the local physics of the reconnection layer (e.g. anomalous resistivity, FLR-effects, or the internal structure of the layer). That is, at any given moment of time, the structure of the MHD equilibrium, i.e. the pressure and current profiles, does not depend on the details of the resistive region solution. However, the reconnection rate at which the global equilibrium states follow each other, is determined by the resistive reconnection region.

The local solution in the reconnection layer depends both on the local physics and on the global solution in terms of the magnetic field $B_{0}$ and the length of the layer $L$.

We found that

- the reconnection should be somewhat faster for the counterhelicity case;

- assuming the Sweet-Parker model for the reconnection layer, the reconnection rate is proportional to the external pushing force $F$ to the 3/4-power (for fixed values of the total poloidal flux $\Psi_{t o t}$, and horizontal size $L_{0}$ ).

Both these results are found to be in a qualitative agreement with the experiments.

\section{APPENDIX: VALIDITY OF THE $B_{w}=$ const - APPROXIMATION.}

Now, let us investigate the question of how good is the $B_{w}=$ const - approximation? i.e. how far do we have to move the wall in order to keep $B_{w}$ fixed?

Since $B_{w}$ and the position of the wall $x_{w}$ are related via

$$
B_{w}=\frac{\Psi_{v a c}}{x_{w}\left(\Psi_{s}\right)-d\left(\Psi_{s}\right)},
$$

the required ( for $B_{w}=$ const) change in $x_{w}$ is equal to the change in $d$.

Initially, the distance $d$ between the magnetic axis and the plasma-vacuum boundary is $d\left(\Psi_{s}=\Psi_{t o t}\right)=a_{0}$. With time, $d$ changes: if a flux layer of width $\Delta x$ is reconnected, its width after the reconnection is $\Delta x^{\prime}=\delta(\theta) \Delta x$, and the corresponding change in $d$, and, therefore, in $x_{w}$, can be written as $\Delta x^{\prime}-\Delta x=(\delta(\theta)-1) \Delta x$. 
Then, the total change in $d$ as function of $\Psi_{s}$ is $\Delta d\left(\Psi_{s}\right) \equiv d\left(\Psi_{s}\right)-a_{0}=\int_{x=a\left(\Psi_{s}\right)}^{x=a_{0}}(\delta(\theta)-$ 1) $d x$. But $\theta=B_{0} / B_{w}=a / a_{0}$, then

$$
\Delta x_{w}\left(\Psi_{s}\right)=\Delta d\left(\Psi_{s}\right) \equiv d\left(\Psi_{s}\right)-a_{0}=a_{0} \int_{\theta\left(\Psi_{s}\right)}^{\theta=1}(\delta(\theta)-1) d \theta
$$

We, however, are more interested in the real displacement (i.e. the displacement in the laboratory frame) of the wall, and, hence, of the plasma-vacuum boundary, which are different from (A2), because the magnetic axis itself is moving. Let $D\left(\Psi_{s}\right)$ be the distance between the midplane and the plasma-vacuum boundary, i.e. $D\left(\Psi_{s}\right)=a\left(\Psi_{s}\right)+d\left(\Psi_{s}\right)$, and let $X_{w}$ be the position of the boundary with respect to the midplane, i.e. $X_{w}=x_{w}+a$. Then, our $B_{w}=$ const condition requires that

$$
\Delta X_{w}\left(\Psi_{s}\right)=\Delta D\left(\Psi_{s}\right)
$$

Then, similar to Eq. (A2), we can write:

$$
\Delta X_{w}\left(\Psi_{s}\right)=\Delta D\left(\Psi_{s}\right) \equiv D\left(\Psi_{s}\right)-D\left(\Psi_{t o t}\right)=a_{0} \int_{\theta\left(\Psi_{s}\right)}^{\theta=1}(\delta(\theta)-2) d \theta
$$

Since $\delta(\theta)<2$ all the time, $\Delta X_{w}, \Delta D<0$, which means that the boundary moves downward, so that external forces (the wall) perform work upon the plasma.

The integral in (A4) can be easily calculated for both co, and counterhelicity cases:

$$
\Delta X_{w}^{c o}\left(\Psi_{s}\right)=\Delta D^{c o}\left(\Psi_{s}\right)=\left.a_{0}\left[-1.2 \theta+\frac{1}{10}\left(\theta \sqrt{36-15 \theta^{2}}+\frac{36}{\sqrt{15}} \arcsin \frac{\sqrt{15} \theta}{6}\right)\right]\right|_{\theta} ^{1}
$$

and

$$
\begin{aligned}
\Delta X_{w}^{\text {counter }}\left(\Psi_{s}\right)= & \Delta D^{\text {counter }}\left(\Psi_{s}\right)=a_{0}\left[-1.2 \theta+\frac{1}{10}\left(\theta \sqrt{16+5 \theta^{2}}+\right.\right. \\
& \left.\left.\frac{16}{\sqrt{5}} \log \left(\sqrt{5} \theta+\sqrt{16+5 \theta^{2}}\right)\right)\right]\left.\right|_{\theta} ^{1},
\end{aligned}
$$

The total displacements of the wall and of the plasma-vacuum boundary in the co and counterhelicity cases are

$$
\Delta X_{w, \text { tot }}^{c o}=\Delta D_{\text {tot }}^{c o}=\Delta D^{c o}(\theta=0) \simeq-0.1 a_{0}
$$


and

$$
\Delta X_{w, \text { tot }}^{c o}=\Delta D_{\text {tot }}^{\text {counter }}=\Delta D^{\text {counter }}(\theta=0) \simeq-0.36 a_{0}
$$

As we see, the displacement of the plasma-vacuum boundary (and, therefore, the required displacement of the wall) can be considered small compared to $a_{0}$, and hence $B_{w}=$ const can be a reasonable approximation even for the situation when the position of the wall is fixed.

\section{ACKNOWLEDGEMENTS.}

This paper benefited considerably from many valuable discussions with Neil Pomphrey who suggested some of the critical ideas. We are also grateful for helpful comments from Wonchull Park, Hantao Ji, and Victoria Dorman.

This paper was prepared under the auspices of the Department of Energy Contract No. DE-AC02-76-CHO-3073. It was also supported by the National Science Foundation under Grants Nos. AST9121847 and ATM-911-4924, and by NASA's Astrophysical program under Grant No. NAGW-2419.

\section{REFERENCES.}

1. M. Yamada, Y. Ono, A. Hayakawa, M. Katsurai, Phys. Review Letters, 65, No.6, p. $721,1990$.

2. M. Yamada, F.W. Perkins, A.K. MacAulay, Y. Ono, and M. Katsurai, Phys. Fluids B, 3 (8), p. 2379, 1991.

3. Y. Ono, A. Morita, M. Katsurai, and M. Yamada, Phys. Fluids B, 5, No.10, p. 3691, 1993.

4. R.L. Stenzel and W. Gekelman, J. Geophys. Res., 86, p.649, 1981.

5. M. Yamada, in "Physics of the Magnetosphere" (ed. B.Sonnerup and P.Song), p. 215, AGU Monograph, 1995.

6. R.M. Kulsrud, Phys. Plasmas, 2(5), p. 1735, 1995. 
7. F. Dahlgren, M. Yamada, F.W.Perkins, N. Pomphrey, Y. Ono, M. Katsurai, Bull. of APS, DPP, 39, No.7, 1Q5, p. 1526, 1994.

8. H. Goldstein "Classical Mechanics" Second ed. Addison-Wesley Pub. co., Reading Mass., 1980

9. V.M. Vasyliunas, Rev. of Geophysics and Space Physics, 13, No.1, p. 303, 1975.

10. D. Biskamp, Phys. Reports, 237, No.4, p. 179, 1994.

11. P.A. Sweet, Electromagnetic Phenomena in Cosmical Physics (ed. by B.Lehnert), p. 123, Cambridge University Press, 1958.

12. E.N. Parker, Astrophysical Journal Supplement Series, 8, p. 177, 1963.

13. H.P. Petschek, AAS-NASA Symposium on Solar Flares, NASA SP50, p.425, 1964.

14. E.R. Priest and T.G. Forbes, J. Geophys. Res.,91, No.A5, p.5579,1986.

15. B.U. Sonnerup, J. Plasma Physics, 4, p. 161, 1970.

16. D. Biskamp, Phys. Fluids, 29, No.5, p. 1520, 1986.

17. T. Tajima and J.-I. Sahai, IEEE Trans. Plasma Sci. PS14, p. 929, 1986. 


\section{F I G U R E C A P T I O N S.}

FIG. 1a. Schematic drawing of the two merging spheromaks at $t=0$.

FIG. 1b. Schematic drawing of the two merging spheromaks at $t>0$. Region $\mathrm{I}$ is the unreconnected flux region, and region II is the common flux region.

FIG. 2. 3-D structure of the magnetic field lines for the co and counterhelicity cases.

FIG. 3. Transition from 2-D geometry to 1-D geometry.

FIG. 4. Illustration for the calculation of the energy change and the work done.

FIG. 5. Plot of the compression factor $\delta$ at the instant of reconnection as a function of $\Psi_{s}$ for the cohelicity and counterhelicity cases. Arrows indicate the direction of the evolution of the reconnection. The actual compression of plasma is $2 / \delta$.

FIG. 6. The normalized pressure in region II $\beta(\Psi)$. At the end of the process, the portion of the magnetic energy converted into the plasma thermal energy goes to 0 in the cohelicity case, and to 1 in the counterhelicity case.

FIG. 7. Common flux ratio $\alpha \equiv \frac{\Psi_{c o m}}{\Psi_{\text {tot }}}=1-\frac{\Psi_{s}}{\Psi_{\text {tot }}}$ as a function of time $t$. As we see, in the counterhelicity case, the reconnection goes somewhat faster at the end of the process, though the beginning is the same.

FIG. 8. Trajectories in the $\left(\Psi_{s}, X_{w}\right)$-space for several different regimes of the evolution of the boundary conditions: trajectory (a) corresponds to $X_{w}=$ const, and trajectories (b) and $\left(\mathrm{b}^{\prime}\right)$ correspond to $B_{w}=$ const in the co and counterhelicity cases, respectively. $\Delta X_{w}$ is the actual displacement of the wall. 


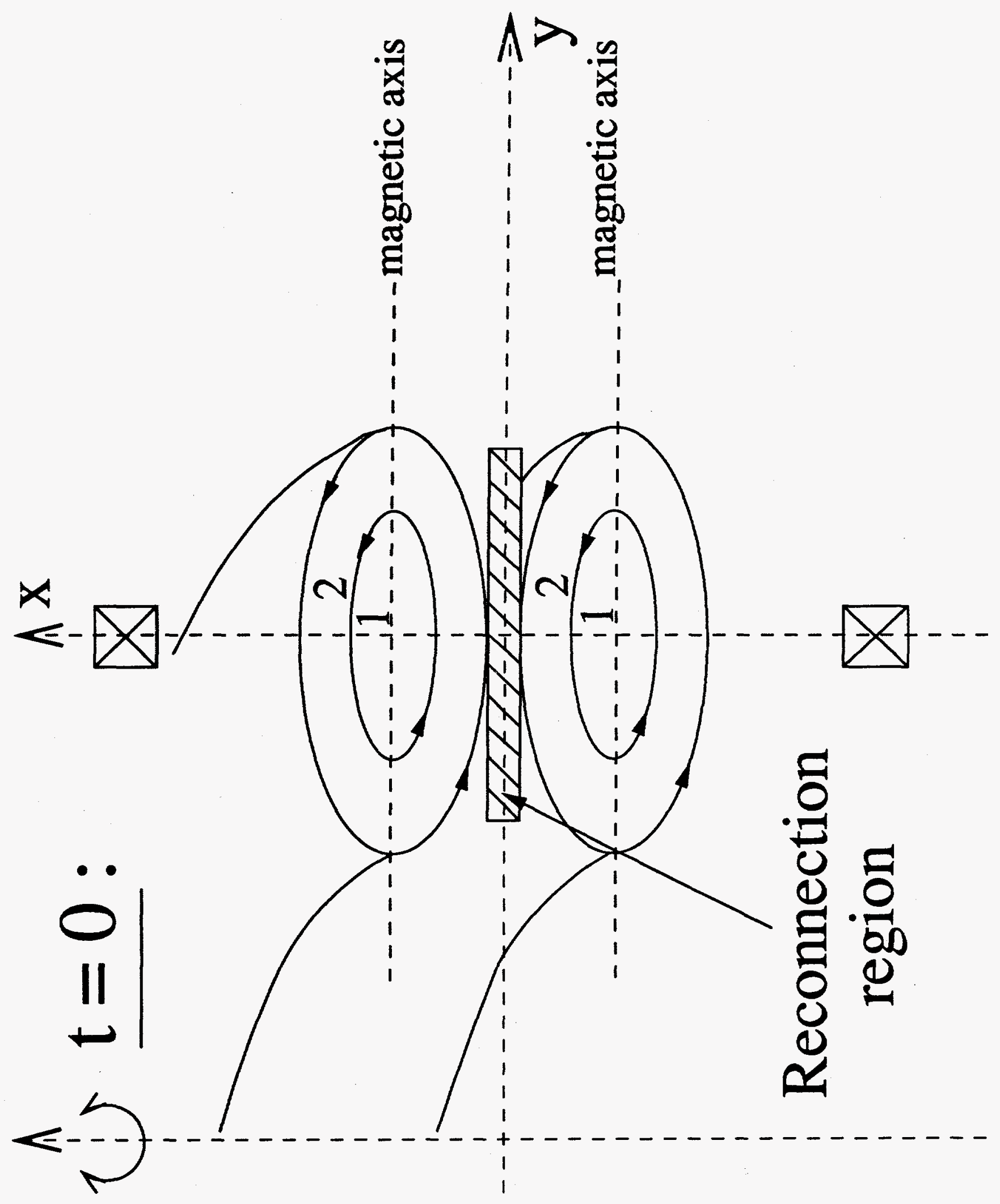

FIG. 1 a. 


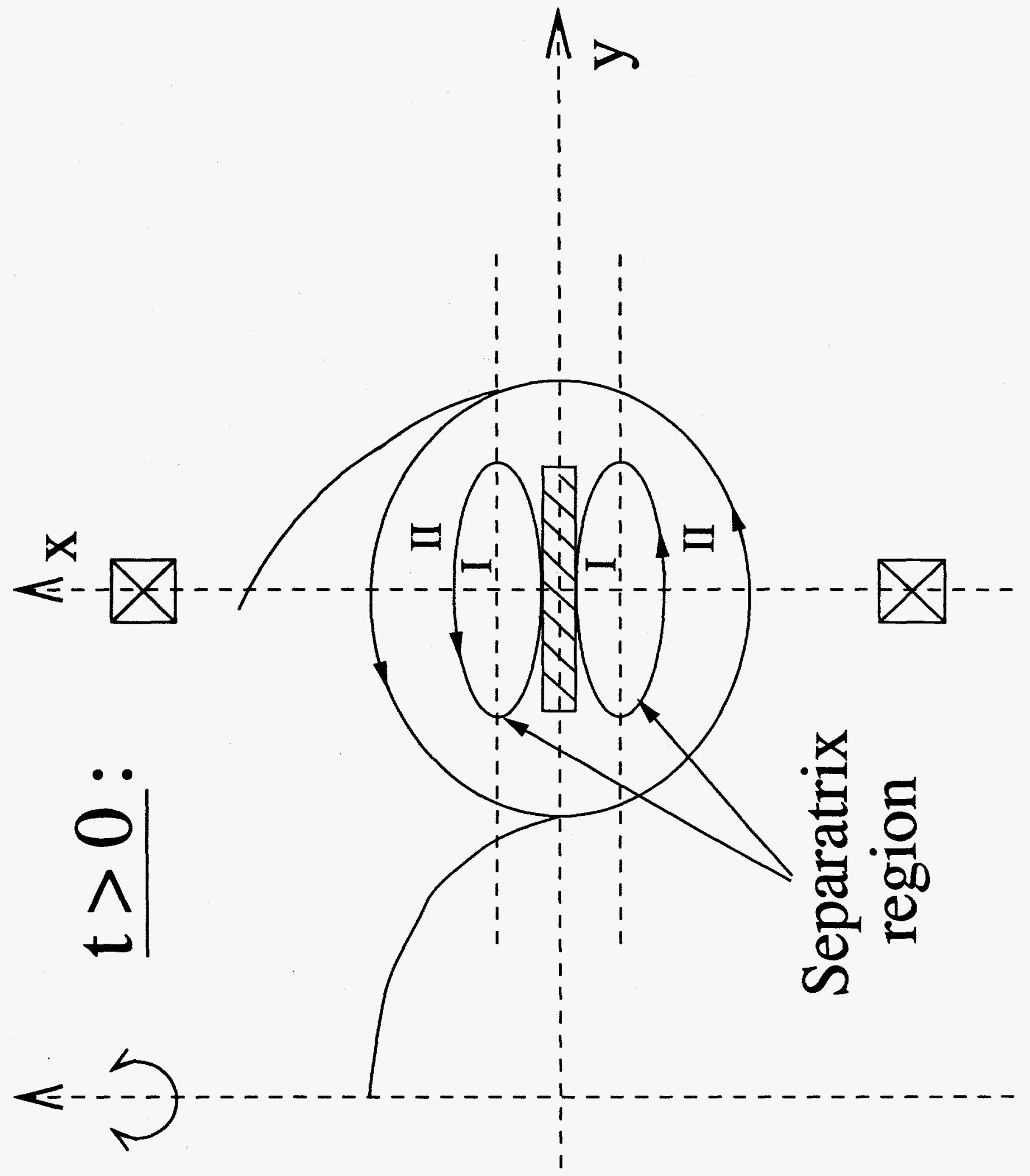

FIG. 1 b. 
' 2 DIA
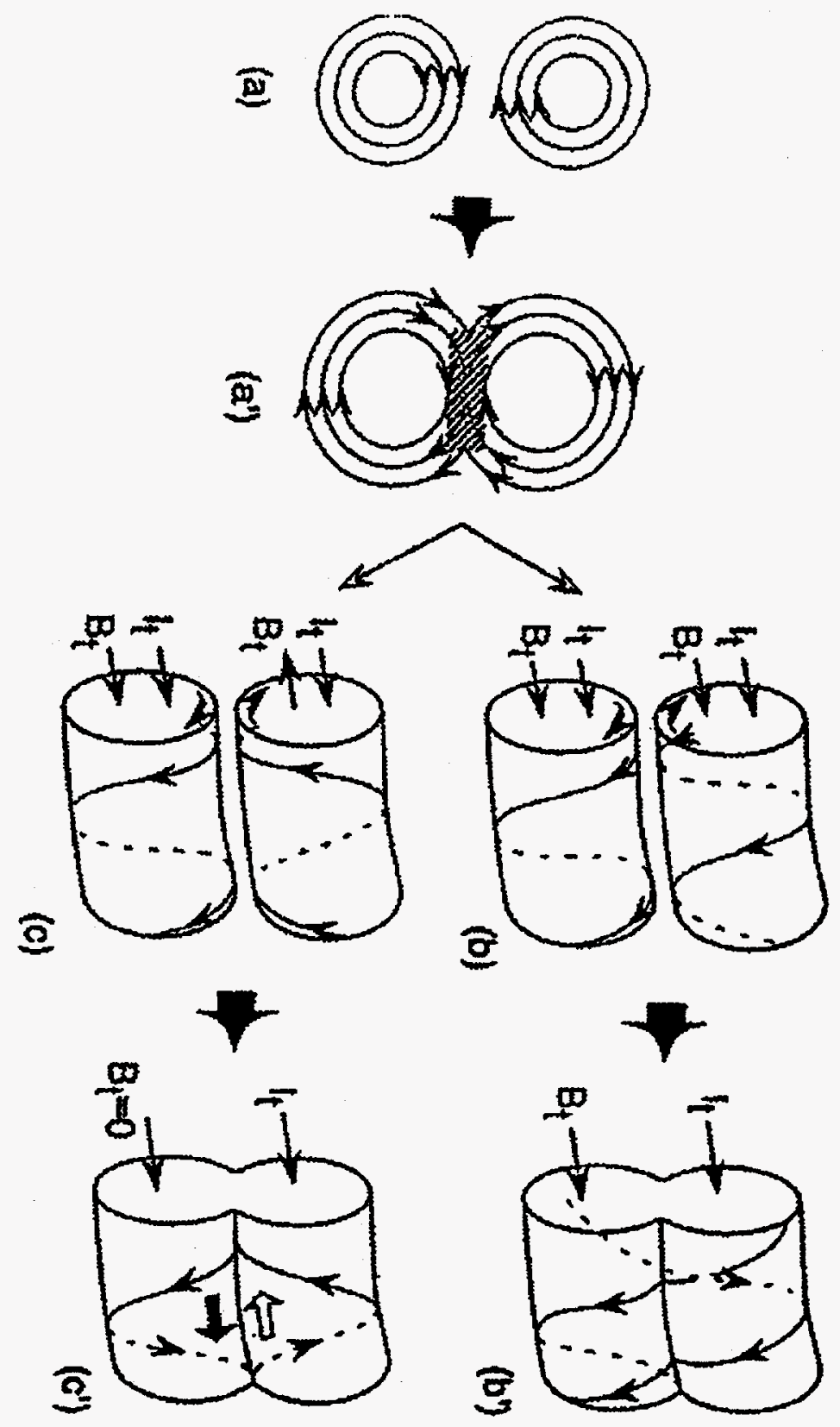

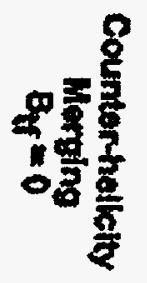

4
4
$2 \frac{9}{3}$
0 


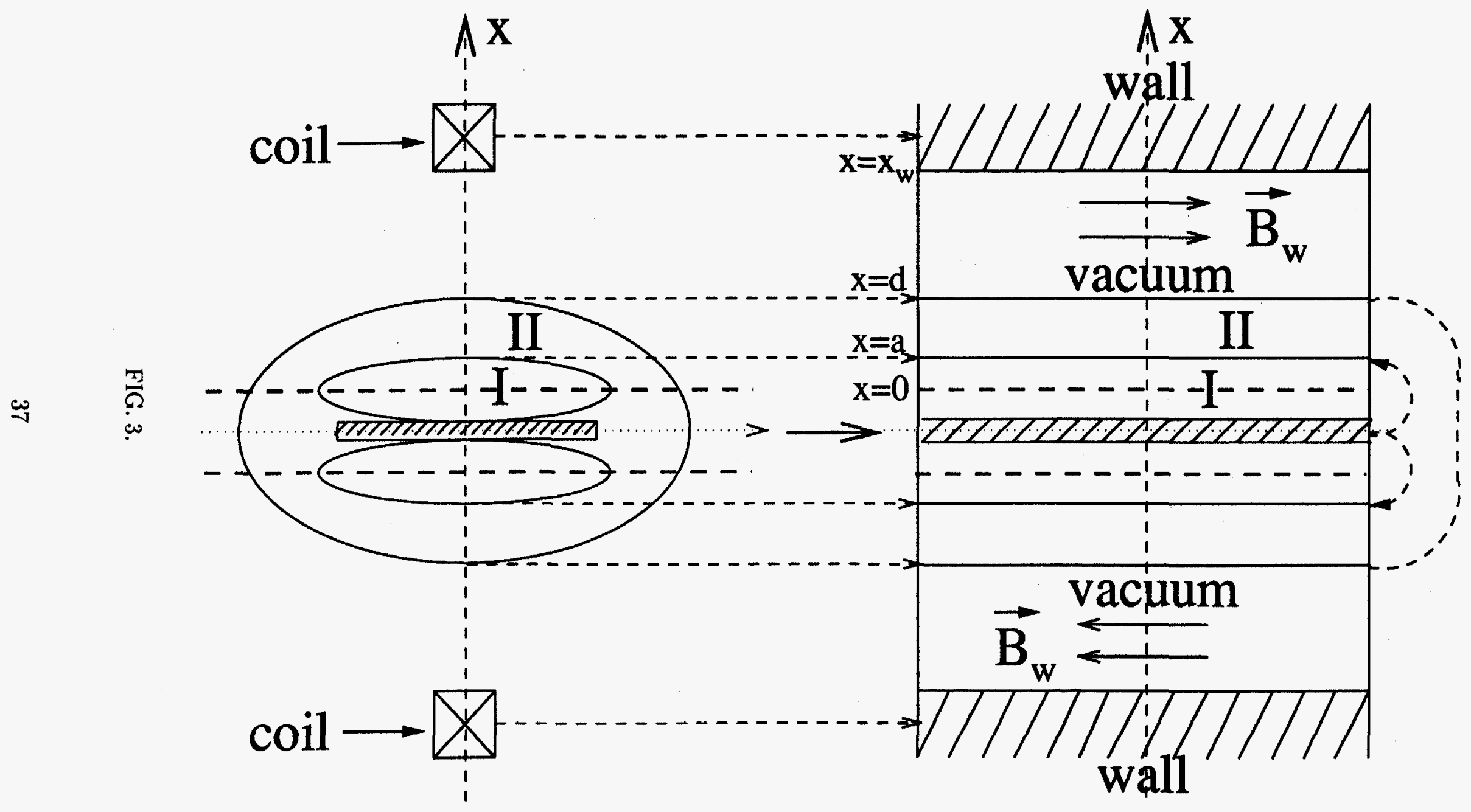




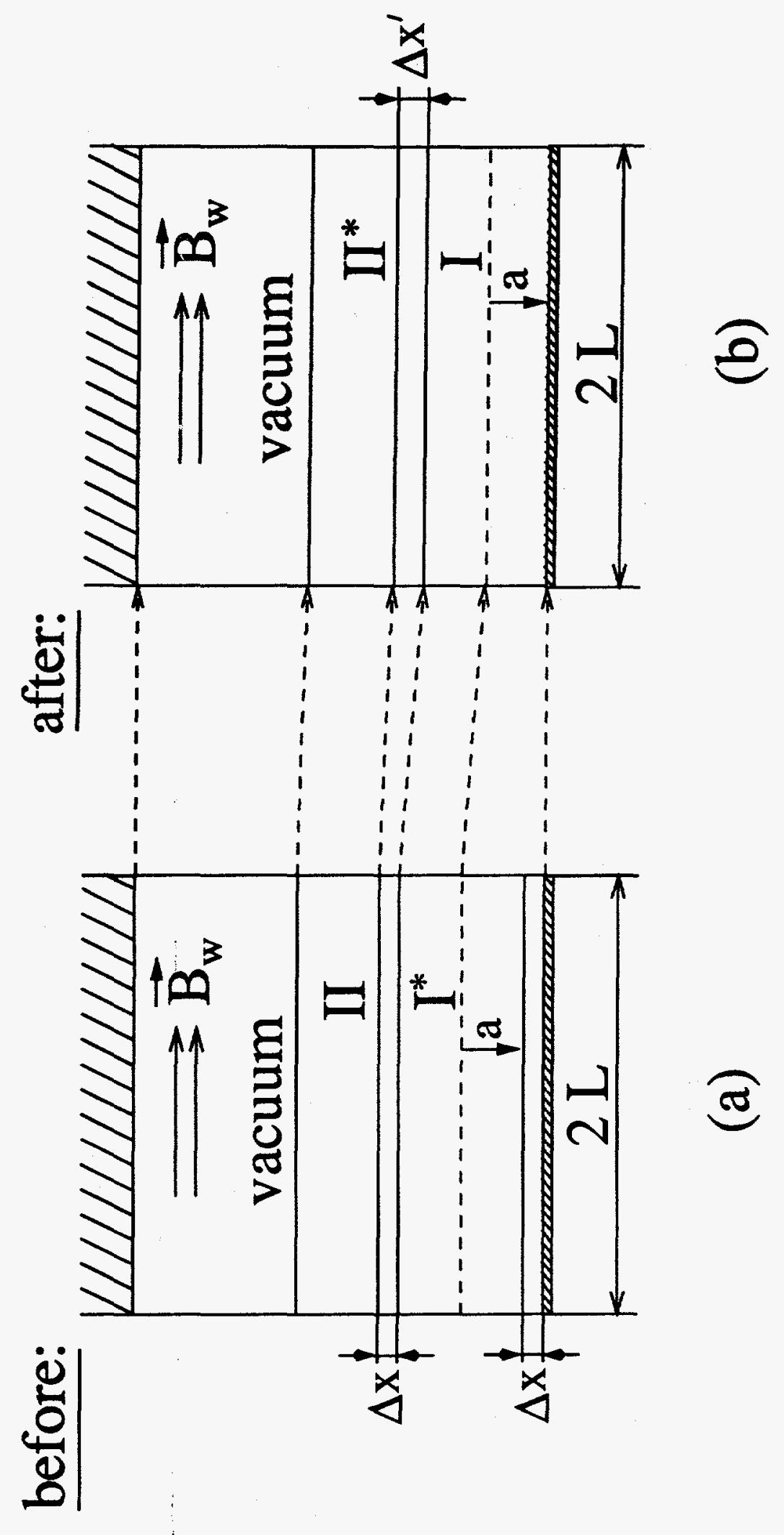

FIG. 4. 


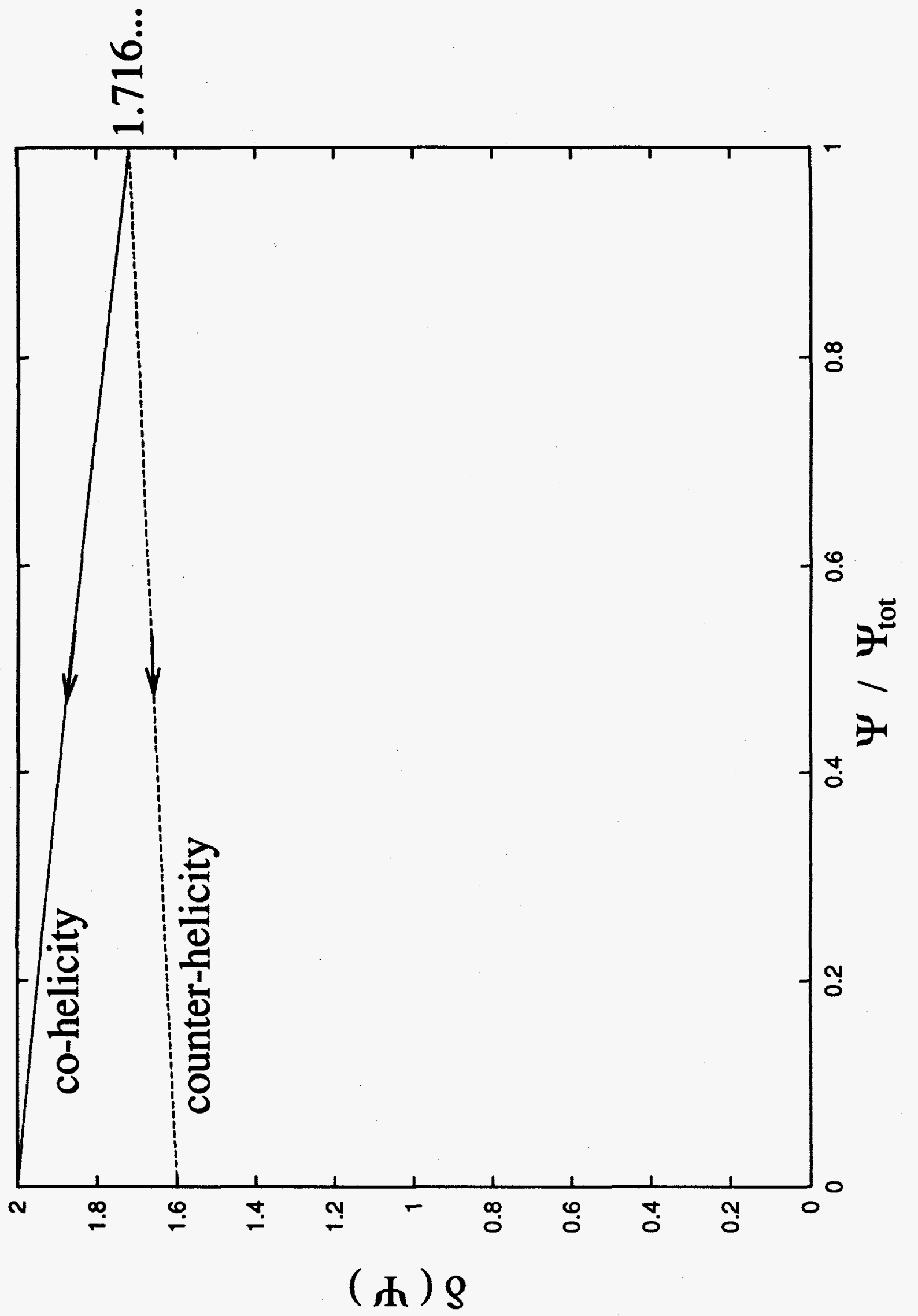

FIG. 5. 


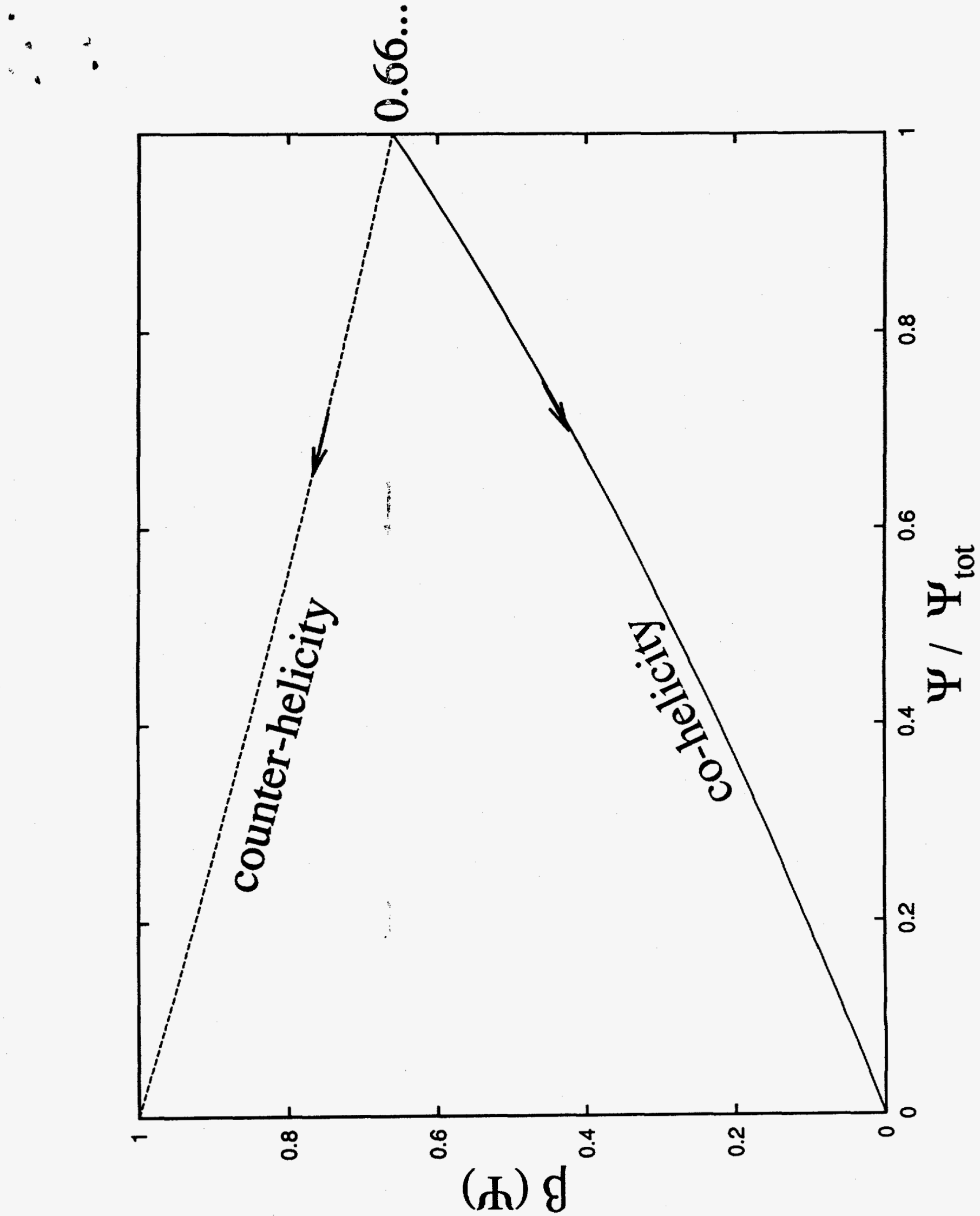

FIG. 6. 


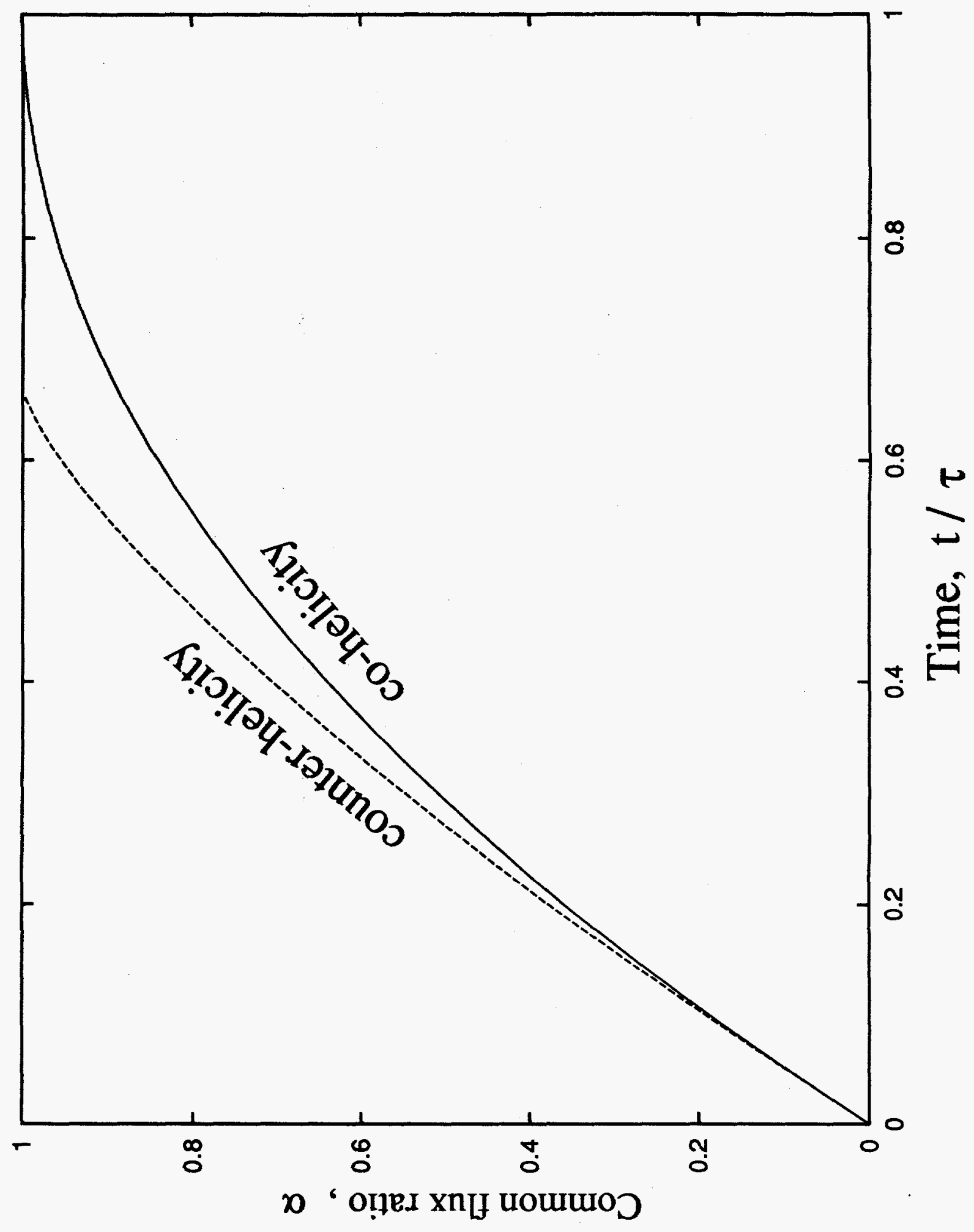

FIG. 7. 


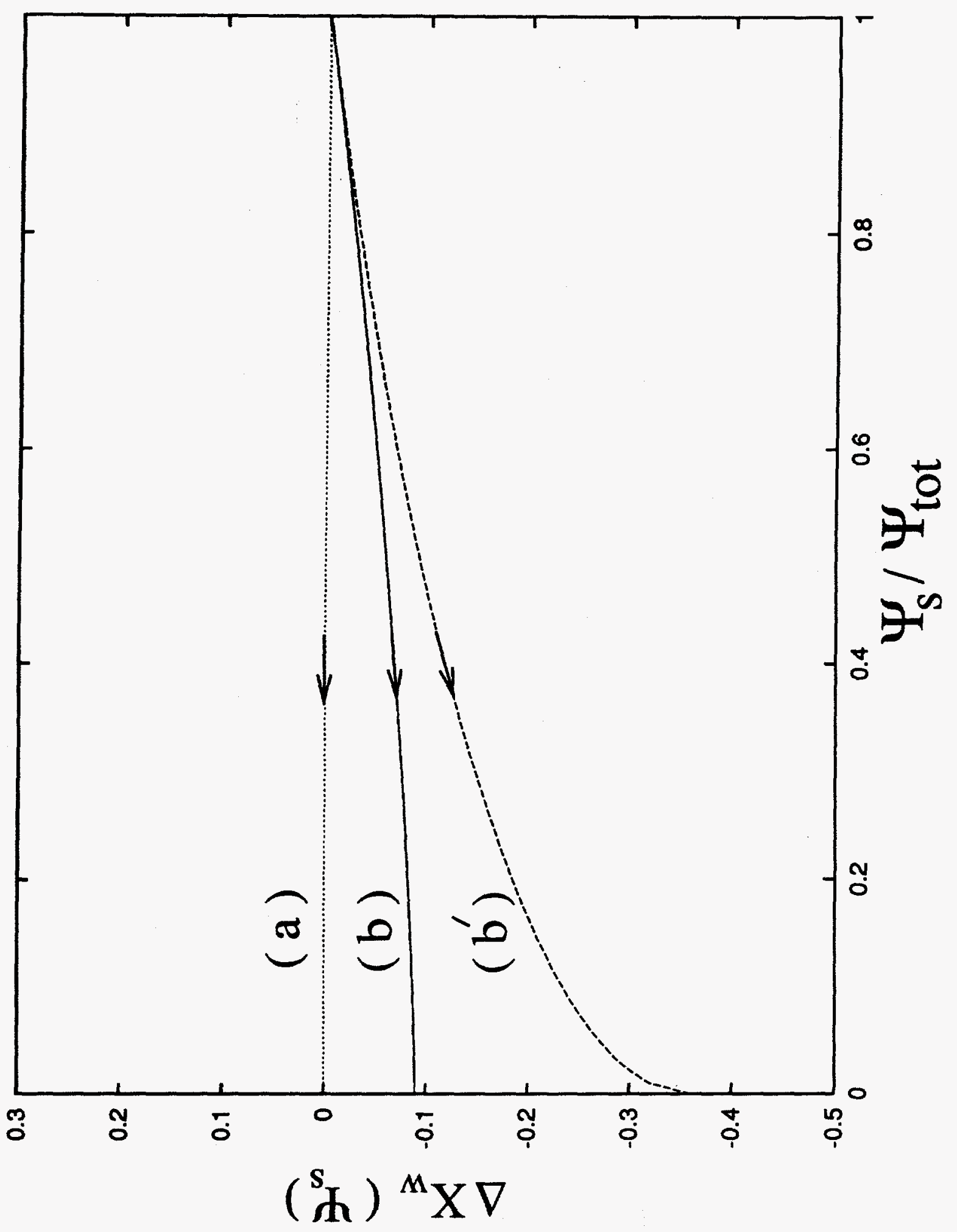

FIG. 8 . 


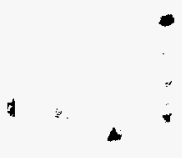

